\title{
Calculation of the local current density in high-temperature superconducting insulated rare earth-barium-copper oxide coils using a volume integral formulation and its contribution to coil protection
}

\author{
Rozier Blandine $^{1}\left(\mathbb{B}\right.$, Badel Arnaud $^{2}$ (1), Ramdane Brahim ${ }^{1}$ and \\ Meunier Gérard ${ }^{1}$ \\ ${ }^{1}$ Université Grenoble Alpes, CNRS, Grenoble INP, G2Elab, F-38000 Grenoble, France \\ ${ }^{2}$ High Field Laboratory for Superconducting Materials, IMR, Tohoku University, Sendai 980-8577, Japan \\ E-mail: blandine.rozier@g2elab.grenoble-inp.fr
}

\begin{abstract}
High-temperature superconducting materials have remarkable current-carrying capabilities, even when operated under high magnetic fields. Since long rare earth-barium-copper oxide (REBCO)-coated conductors are now available (thanks to improvements in the fabrication process) this material has become an attractive option for high field magnet applications. However, such extreme operating conditions require an efficient quench protection system to prevent the coil from developing damaging hot spots, which greatly depends on the winding used. We focus here on the protection of insulated HTS coils against thermal runaways that can locally destroy the magnet. We developed a transient two-dimensional (2D) axisymmetric model using a volume integral formulation based on generalization of the partial element equivalent circuit method to compute the local current density distribution inside REBCO-insulated coils and account for local performance variations. Indeed, the most interesting property of integral methods is the requirement that only active regions are meshed, which leads to a significant reduction in the size of the problem. The formulation is introduced for general 3D cases and its adaptation to 2D axisymmetric problems is detailed. The formulation has been validated thanks to a bulk magnetization benchmark, the results of which (obtained with the finite element method) were compared with our integral formulation solution. The model has also been compared with experimental data obtained on a double pancake coil. The objective is to study the effects of magnetization on the transient voltage due to dynamic current distribution when ramping up the magnet so as to be able to determine some key parameters associated with coil protection. Such an approach is developed on a small-scale test case and the transient behaviours observed are discussed.
\end{abstract}

Keywords: volume integral method, quench protection, insulated HTS coils, current distribution 


\section{Introduction}

Superconducting materials are widely used for applications in magnetic field generation as they are able to carry current without any dissipation under specific operating conditions. Low-temperature superconducting (LTS) materials such as $\mathrm{NbTi}$ or $\mathrm{Nb}_{3} \mathrm{Sn}$ are classically used, but due to the decrease in their current-carrying capabilities with increase in magnetic field and temperature they cannot generate fields higher than $22 \mathrm{~T}$, and only then if maintained at a very low temperature [1].

Due to better performance in terms of both critical current and critical field $\left(\mathrm{H}_{\mathrm{c} 2}\right)$, high-temperature superconducting (HTS) magnets are in principle capable of reaching a much higher field, making them a cost-effective alternative to resistive magnets for very high magnetic field generation [2].

Several projects have been carried out recently with the aim of developing high field magnets realized partially or totally with rare earth-barium-copper oxide (REBCO) tapes . In France, the National High Field Magnet Laboratory is about to test a $10 \mathrm{~T}$ HTS insert into an already existing resistive magnet generating $20 \mathrm{~T}$ so as to test its ability to operate with $30 \mathrm{~T}$ in its centre [3]. A full superconducting $32 \mathrm{~T}$ magnet has been developed at the National High Magnetic Field Laboratory at Tallahassee, USA [4, 5]. This hybrid LTS-HTS magnet is composed of a LTS outsert generating $15 \mathrm{~T}$ and a HTS insert made of a set of double pancake coils generating the additional $17 \mathrm{~T}$. Recently tested, it has achieved the highest magnetic field generated by a fully superconducting magnet to date, with a central field value of $27 \mathrm{~T}$ [6]. At the same time, another project developed by the Chinese Academy of Science aims to design a $25 \mathrm{~T}$ superconducting magnet made of $\mathrm{NbTi}$ and $\mathrm{Nb}_{3} \mathrm{Sn}$ coils and a REBCO insert at the Extreme Condition Experimental Science Facility (ExCES) for studying the properties of condensed matter [7-9]. In Japan, the High Field Laboratory for Superconducting Materials at Tohoku University has developed a $25 \mathrm{~T}$ cryogen-free superconducting magnet with a $11.5 \mathrm{~T}$ REBCO insert, which was later replaced by an equivalent BSCCO insert [10].

Although these magnets exhibit very promising performance and an improved fabrication process able to produce sufficiently long REBCO tapes, HTS magnets are not yet very widespread. Some key points are still being studied, especially with regard to their behaviour close to their critical current, particularly when operating under a very high current density. HTS coil protection strategies are of prime importance to ensure safe operating conditions, and have to be chosen carefully depending on the specificities of each magnet, such as its winding principle, which will greatly influence the final protection design.

In the past few years new winding techniques have emerged to deal with protection issues. In non-insulated coils, the insulation is removed in order to allow the current to flow from one turn to the next in case of initiation of a local transition, thus bypassing the dissipative regions. Highly thermally stable, they may recover after a quench without any additional protection system $[11,12]$. Another alternative is to use a metal-as-insulation winding, in which the coil is cowound with a metallic layer to increase the turn-to-turn contact resistance. Nearly self-protection behaviour has also been reported for this kind of coil [13]. The main drawback of these designs is their slower dynamics, which may be an issue in some applications.

More classical insulated coils always require an efficient active protection system, like heaters as in [5] or the use of a dump resistance as in [10]. In this work, only the case of insulated coils is considered.

Quench ignition is characterized by a rapid increase in voltage, meaning that quench detection techniques are usually based on monitoring the coil voltage. Because of inhomogeneities of the critical current density $\mathrm{J}_{\mathrm{c}}$ along the tape associated with slow normal zone propagation velocities (a few $\mathrm{cm} \mathrm{s}^{-1}$ ) [14, 15], early detection is necessary to prevent the coil from damaging hot spots [16]. The main issue with this strategy is the requirement to detect a small variation of the voltage signal (in this case the dissipative component of the total voltage) that is embedded in a much higher-magnitude signal (the transient component due to the current variation when ramping up the coil) which is also very noisy due to the coil's large inductance.

In order to make the voltage measurement clearer, it is possible to add compensation coils so as to compensate the inductive behaviour of the HTS coil and obtain more accurate measurements [17]. However, this solution does not perfectly cancel the inductive voltage of the HTS coil, as the compensation coils have a constant inductance value while the actual inductance of the HTS coil depends on the current density distribution in the conductor, which evolves with time [18]. Early quench detection might become difficult if the transient voltage due to inductance variations is of the same order of magnitude as the desired voltage threshold value.

Another major issue is the magnetization phenomenon: current distribution greatly depends on the operating conditions previously experienced by the coil. For instance, when ramping up the coil, discharging it and then ramping it up higher, a fast increasing voltage will appear when the previous maximum current is overcome. This transient behaviour is only due to current redistribution and must not be confused with the appearance of a dissipative zone.

Modelling of magnetization currents in REBCO coils is a problem that has been widely studied in superconducting applications, especially for computation of AC losses [19-23] as they have a significant impact on the quality of the magnetic field or the refrigeration requirements for instance. However, we propose here to investigate these transient behaviours for protection purposes. We thus developed a numerical model using a volume integral formulation in order to evaluate the transient behaviour of insulated REBCO coils submitted to successive current ramps.

The paper is organized as follows. Firstly, the general volume integral formulation coupled to a circuit approach is presented, and its adaptation to two-dimensional (2D) axisymmetric problems is detailed. Next, the model is applied to a benchmark test case dealing with the HTS bulk magnetization problem for validation. A sensitivity analysis is then 
done, followed by a comparison of the simulation results with experimental data obtained on a double pancake coil tested at low temperature and self-field conditions. Finally, an application of the model is presented for a small-scale test case and results regarding protection are discussed.

\section{J-formulation presentation}

Analytical models can be derived from constitutive equations to compute the transient behaviour of superconducting applications under specific conditions and on simple geometries such as tapes [24, 25] or cylinders [26]. However, the critical state model [27] used as constitutive law in analytical models is not very well adapted to HTS materials which start to dissipate before the current density reaches $\mathrm{J}_{\mathrm{c}}$ because they exhibit a smoother transition from superconducting to normal states. Besides, the variation of $\mathrm{J}_{\mathrm{c}}$ with magnetic field and orientation cannot be taken into account in analytical models. Therefore, modelling of complex geometries and/or complex constitutive laws accounting for physical properties (variation with the magnetic field, the temperature, etc) require the use of numerical methods. Of all the numerical tools available, the most widespread method for electromagnetic computations is certainly the finite element method (FEM), which has already been successfully applied to many superconducting problems [28]. However, depending on the formulation chosen, some important issues can arise: current conservation which is not strongly ensured, the high aspect ratio of HTS tapes which requires an extensive mesh for the nonlinear algorithm to converge, and so on. We propose an original approach using a volume integral formulation based on a generalization of the partial element equivalent circuit method to compute the local current density distribution inside a HTS magnet while taking into account the effects of local performance variations.

\subsection{Volume integral methods}

Volume integral methods (VIMs) started to be seriously considered and developed for electromagnetic computations after the introduction in the 1980s [29] of first- and secondorder Whitney elements, also known as edge and facet elements, respectively. Their intrinsic properties are perfectly suited to $\mathbf{H}$ and $\mathbf{E}$ field representation as edge elements conserve the tangential component, and to $\mathbf{B}$ and $\mathbf{J}$ fields for facet elements whose conservation of the normal component is ensured by definition. However, the main issue when using such an approach is the generation of full matrices, instead of sparse ones as for the FEM (the latter being much more convenient for storage and computations). Indeed, integral methods account for all interactions of elements of the domain on each other, which also results in better approximations than considering only the closest interactions as the FEM does. Nevertheless, this class of methods is of interest again with the development of new powerful numerical for tools dealing with matrix compression [30] and issues of full matrix integration. The main advantage of using VIMs over the FEM is that only active regions need to be discretized: no mesh is required for air regions, which consequently reduces the final problem size. Although superconductor modelling mostly refers to the FEM, variational principles, which share with VIMs the advantage of meshing active regions only, have already been used successfully for 2D [31-35] and 3D $[36,37]$ superconducting problems.

The J-formulation for modelling superconducting problems can be quite convenient as the current conservation condition is automatically enforced, contrary to the $\mathrm{H}$-formulation (FEM) which requires some sophisticated modifications to avoid current diffusing to the air [38]. The formulation presented here is based on a generalization of the partial element equivalent circuit method (the PEEC method) [39] which is already used for computation of the current distribution inside superconducting coils [40]. It combines the circuit approach (based on Kirchhoff's law) with a magnetic field problem to be solved on a physical domain (with a specific meshed geometry) as impedances of the equivalent electrical circuit are dependent on magnetic field. The J-formulation enables automatic generation of the equivalent electrical circuit from any meshed physical domain thanks to the use of facet elements for the interpolation of current density. Each node of the equivalent electrical circuit is defined by the barycentre of each element. Therefore, connections of the physical domain to external circuit branches are particularly easy to implement.

\subsection{Continuous equation}

An arbitrarily shaped superconducting domain is considered. The magneto-quasistatic approximation is assumed, meaning no displacement currents are considered and the divergencefree current density condition has to be satisfied.

From Maxwell's equations, the electric field $\mathbf{E}$ can be expressed anywhere in the domain by

$$
\mathbf{E}=-\frac{\partial \mathbf{A}}{\partial \mathrm{t}}-\nabla \mathrm{V}
$$

where A represents the magnetic vector potential and $\mathrm{V}$ is the electric scalar potential. The constitutive law of any superconducting material is such that

$$
\mathbf{E}=\rho(\mathrm{T}, \mathbf{B}, \mathbf{J}) \mathbf{J}
$$

where $\rho$ is the electrical resistivity, which depends on the temperature $\mathrm{T}$, the magnetic flux density $\mathbf{B}$ and the current density $\mathbf{J}$. On the other hand, the magnetic vector potential A can be divided into two different terms, depending on its origin: an $\mathbf{A}_{0}$ vector potential due to a background magnetic field, which can be uniform or not, and an $\mathbf{A}_{\mathrm{sc}}$ vector potential induced by the current flowing through the superconducting device

$$
\mathbf{A}=\mathbf{A}_{0}+\mathbf{A}_{\mathrm{sc}}(\mathbf{J}) \text {. }
$$


As superconductors have no magnetic properties, the magnetic vector potential originating from the physical domain, $\mathbf{A}_{\mathrm{sc}}$, can be computed at any point using the 3D BiotSavart law

$$
\mathbf{A}_{\mathrm{sc}}(\mathrm{Q})_{\mathrm{P}}=\frac{\mu_{0}}{4 \pi} \int_{\Omega_{\mathrm{c}}} \frac{\mathbf{J}}{\mathrm{PQ}} \mathrm{d} \Omega_{\mathrm{c}}=\frac{\mu_{0}}{4 \pi} \int_{\Omega_{\mathrm{c}}} \frac{\mathbf{J}}{\mathbf{r}} \mathrm{d} \Omega_{\mathrm{c}}
$$

where $\mu_{0}$ represents the vacuum permeability, $\mathrm{P}$ is the source point, $\mathrm{Q}$ is the field point ( $\mathbf{r}$ is the vector from the source point to the field point) and $\Omega_{\mathrm{c}}$ is the conducting domain. The $\mathrm{J}$-formulation is then derived from equations (2)-(4):

$$
\rho(\mathrm{T}, \mathbf{B}, \mathbf{J}) \mathbf{J}+\frac{\mu_{0}}{4 \pi} \frac{\partial}{\partial \mathrm{t}} \int_{\Omega_{\mathrm{c}}} \frac{\mathbf{J}}{\mathbf{r}} \mathrm{d} \Omega_{\mathrm{c}}=-\frac{\partial \mathbf{A}_{0}}{\partial \mathrm{t}}-\nabla \mathrm{V} .
$$

\subsection{Discretization and circuit coupling}

Equation (5) cannot be solved in its continuous form but it can be approximated using numerical methods. A mesh is generated based on the geometry of the physical domain. The unknown $\mathbf{J}$ is interpolated with facet shape functions as follows (the sum is taken over all the elements constituting the mesh)

$$
\mathbf{J}=\sum_{\mathrm{k}} \mathbf{w}_{\mathrm{fk}} \mathrm{I}_{\mathrm{k}}
$$

where $\mathbf{w}_{\mathrm{fk}}$ is the shape function associated with the $\mathrm{kth}$ facet of the mesh and $\mathrm{I}_{\mathrm{k}}$ is the current flux through this facet. Therefore, degrees of freedom (DoF) are related to the facets of the finite element mesh. Facet shape functions have been computed for various types of elements such as tetrahedra, hexahedra or prisms [41]. As mentioned in section 2.1, such shape functions are very well suited to divergence-free vector field interpolation as their main property is that the normal component of each shape function $\mathbf{w}_{\mathrm{fk}}$ is conserved through each facet [29]. The conservation properties of first-order facet shape functions are described by equations (7) and (8):

$$
\begin{gathered}
\int_{S_{\mathrm{k}}} \mathbf{w}_{\mathrm{fk}} \cdot \mathbf{n d S}= \pm \frac{1}{S_{\mathrm{k}}} \\
\operatorname{div}\left(\mathbf{w}_{\mathrm{fk}}\right)= \pm \frac{1}{\mathrm{~V}_{\mathrm{fe}}}
\end{gathered}
$$

where $S_{k}$ refers to the surface of facet element $k, \mathbf{n}$ is its normal vector and $\mathrm{V}_{\mathrm{e}}$ corresponds to the volume of the element to which the kth facet belongs. The sign depends on the orientation of the facet element.

In order to convert the continuous operator problem of equation (5) into a discrete problem, the Galerkin method is applied, meaning the weighting functions are chosen as equal to basis functions defined here by first-order facet shape functions. The weak formulation can be written as

$$
\left([\mathrm{R}]+\frac{\partial}{\partial \mathrm{t}}[\mathrm{L}]\right)\left[\mathrm{I}_{\mathrm{B}}\right]=\frac{\partial}{\partial \mathrm{t}}\left[\mathrm{A}_{0}\right]+[\Delta \mathrm{V}]
$$

with

$$
\mathrm{R}_{\mathrm{ij}}=\int_{\Omega_{\mathrm{i}}} \mathbf{w}_{\mathrm{i}} \cdot \rho \cdot \mathbf{w}_{\mathrm{j}} \mathrm{d} \Omega_{\mathrm{i}}
$$

$$
\begin{gathered}
\mathrm{L}_{\mathrm{ij}}=\frac{\mu_{0}}{4 \pi} \int_{\Omega_{\mathrm{i}}} \mathbf{w}_{\mathrm{i}} \int_{\Omega_{\mathrm{c}}} \frac{\mathbf{w}_{\mathrm{j}}}{\mathbf{r}} \mathrm{d} \Omega_{\mathrm{c}} \mathrm{d} \Omega_{\mathrm{i}} \\
\mathrm{A}_{0 \mathrm{i}}=-\int_{\Omega_{\mathrm{i}}} \mathbf{w}_{\mathrm{i}} \cdot \mathbf{A}_{0} \mathrm{~d} \Omega_{\mathrm{i}} \\
\Delta \mathrm{V}_{\mathrm{i}}=-\int_{\Omega_{\mathrm{i}}} \mathbf{w}_{\mathrm{i}} \cdot \nabla \mathrm{Vd} \Omega_{\mathrm{i}} .
\end{gathered}
$$

This matrix expression of the weak formulation enables us to introduce the circuit coupling which results straightforwardly from the use of facet elements. Each term of equation (9) defines an equivalent electrical component that can be interpreted using circuit theory: $\left[\mathrm{I}_{\mathrm{B}}\right]$ is the branch current vector (a branch is related to the equivalent electrical circuit and associated with a facet; it is built based on the dual mesh of the primal mesh, i.e. it connects barycentres of two adjacent elements); [R] refers to the resistance circuit and is called the resistivity matrix; [L] gathers both self and mutual inductances and is referred to as the inductive matrix; vector $[\Delta \mathrm{V}]$ is related to branch voltages and represents the average voltage drop between two adjacent elements sharing the same facet; and vector $\left[\mathrm{A}_{0}\right]$ corresponds to an external magnetic field source, like a surrounding coil for example [32]. It has to be pointed out that the resistivity matrix is by definition a sparse matrix because $R_{i j}$ is non-zero only if facets $i$ and $j$ belong to the same element: the $[R]$ matrix is thus computed thanks to the FEM assembly process. By contrast, the inductive matrix is a full matrix assembly representative of integral methods: each term $\mathrm{L}_{\mathrm{ij}}$ is computed by integrating the 3D Green kernel defined by

$$
\mathrm{G}_{3 \mathrm{D}}(\mathrm{r})=\frac{1}{4 \pi \cdot \mathrm{r}}
$$

The Green kernel integration can be realized by numerical or semi-analytical integration methods for close to very close interactions, and correction techniques are required for self-integration $(\mathrm{r}=0)$ [42]. To improve computational performances, integration of far interactions can be estimated using matrix compression methods such as the fast multipole method [30], the adaptive cross approximation [43] or the hybrid cross approximation [44].

This problem is solved using a circuit solver based on the determination of independent loops adapted to arbitrarily shaped 3D conducting devices [45]. It results in the generation of an incident matrix, [M], computed thanks to the independent loop search algorithm also described in [45] and composed of 0 and \pm 1 values only. The determination of [M] is based on Kirchhoff's circuit laws and has the following properties:

$$
[\mathrm{M}][\Delta \mathrm{V}]=[0] \Leftrightarrow[\mathrm{M}]^{\mathrm{t}}\left[\mathrm{I}_{\mathrm{L}}\right]=\left[\mathrm{I}_{\mathrm{B}}\right]
$$

where $\left[\mathrm{I}_{\mathrm{L}}\right]$ is an independent loops-based current vector defined as a combination of the branch currents $\left[\mathrm{I}_{\mathrm{B}}\right]$, and $[\mathrm{M}]^{\mathrm{t}}$ is the transpose of matrix $[\mathrm{M}]$. Therefore, equation (9) 
becomes

$$
\begin{array}{r}
{[\mathrm{M}]\left([\mathrm{R}]+\frac{\partial}{\partial \mathrm{t}}[\mathrm{L}]\right)[\mathrm{M}]^{\mathrm{t}}\left[\mathrm{I}_{\mathrm{L}}\right]} \\
=[\mathrm{M}]\left(\frac{\partial}{\partial \mathrm{t}}\left[\mathrm{A}_{0}\right]+[\Delta \mathrm{V}]\right) .
\end{array}
$$

If no external branch is connected, a Neumann boundary condition is applied to all external faces of the conducting domain (intrinsic to equation (16)).

\subsection{Voltage-driven devices}

The equivalent electrical circuit approach enables us to add external circuit branches to the main circuit built out of the dual mesh. In this section, a voltage generator is considered, which imposes a voltage constraint on one of the two nodes related to the facets directly connected to that source. To take into account this additional boundary condition, the incident matrix $[\mathrm{M}]$ is divided into two submatrices: $\left[\mathrm{M}_{\mathrm{RL}}\right]$ related to the physical domain and $\left[\mathrm{M}_{\mathrm{S}}\right]$ to account for the additional voltage drop added by the source:

$$
\left[\mathrm{M}_{\mathrm{RL}} \mathrm{M}_{\mathrm{S}}\right]\left[\begin{array}{c}
\Delta \mathrm{V} \\
\Delta \mathrm{V}_{\mathrm{S}}
\end{array}\right]=[0]
$$

where $\Delta \mathrm{V}_{\mathrm{s}}$ is the value of the voltage source. Combining equations (16) and (17) leads to the weak J-formulation adapted to a voltage generator

$$
\begin{aligned}
& {\left[\mathrm{M}_{\mathrm{RL}}\right]\left([\mathrm{R}]+\frac{\partial}{\partial \mathrm{t}}[\mathrm{L}]\right)\left[\mathrm{M}_{\mathrm{RL}}\right]^{\mathrm{t}}\left[\mathrm{I}_{\mathrm{L}}\right]} \\
& =\left[\mathrm{M}_{\mathrm{RL}}\right] \frac{\partial}{\partial \mathrm{t}}\left[\mathrm{A}_{0}\right]-\left[\mathrm{M}_{\mathrm{S}}\right]\left[\Delta \mathrm{V}_{\mathrm{S}}\right]
\end{aligned}
$$

\subsection{Current-driven devices}

Most of the time superconducting magnets are connected to a current generator which imposes a transport current to control the value of the magnetic field. Such a constraint is expressed by

$$
\int_{\mathrm{S}} \mathbf{J} \mathrm{dS}=\sum_{\mathrm{p}} \int_{\mathrm{S}} \mathbf{w}_{\mathrm{fp}} \mathrm{I}_{\mathrm{p}} \mathrm{d} \mathrm{S}=\mathrm{I}_{\mathrm{S}}
$$

where $I_{S}$ is the value of the transport current and the subscript $\mathrm{p}$ refers to all the facets directly connected to the current generator. From the point of view of an electrical circuit, connection of an external current source is straightforward as it just adds a new branch defined by the circuit equation which has to comply with Kirchhoff's circuit laws. Using the same definition of $\left[\mathrm{M}_{\mathrm{RL}}\right]$ and $\left[\mathrm{M}_{\mathrm{S}}\right]$ as in section 2.4 , the constraint expressed in (19) can thus be rewritten in terms of an electrical circuit (see equation (20)), meaning that the sum of the current at each node connected to the external branch must be equal to the transport current value imposed by the source:

$$
\left[\mathrm{M}_{\mathrm{S}}\right]^{\mathrm{t}}\left[\mathrm{I}_{\mathrm{L}}\right]=\left[\mathrm{I}_{\mathrm{S}}\right]
$$

Such an additional constraint (applied on facets directly connected to the source only) is then imposed by combining equations (20) and (18), the latter still being verified for a current-driven problem although the source voltage drop becomes an unknown instead of an imposed value:

$$
\begin{gathered}
\left(\begin{array}{cc}
{\left[\mathrm{M}_{\mathrm{RL}}\right]\left([\mathrm{R}]+\frac{\partial}{\partial \mathrm{t}}[\mathrm{L}]\right)\left[\mathrm{M}_{\mathrm{RL}}\right]^{\mathrm{t}}} & {\left[\mathrm{M}_{\mathrm{S}}\right]} \\
{\left[\mathrm{M}_{\mathrm{S}}\right]^{\mathrm{t}}} & {[0]}
\end{array}\right) \cdot\left(\begin{array}{c}
{\left[\mathrm{I}_{\mathrm{L}}\right]} \\
{\left[\Delta \mathrm{V}_{\mathrm{S}}\right]}
\end{array}\right) \\
=\left(\begin{array}{c}
{\left[\mathrm{M}_{\mathrm{RL}}\right] \frac{\partial}{\partial \mathrm{t}}\left[\mathrm{A}_{0}\right]} \\
{\left[\mathrm{I}_{\mathrm{S}}\right]}
\end{array}\right) .
\end{gathered}
$$

\section{Numerical modelling of HTS coils using the J-formulation}

\subsection{Coil modelling: from $3 D$ to $2 D$}

The objective is to compute the current distribution inside an insulated REBCO coil. We consider here an axisymmetric problem, which means that the source must generate a perfectly axisymmetric field, as in the case of a surrounding cylinder, where the magnetic field is oriented along the z-direction only, or at least the source complies with a circular approximation so as to extend it to closely packed coils which are actually spirals [37, 38]. Thus the current density $\mathbf{J}$ flows perpendicularly to the cross-section and the vector potential $\mathbf{A}$ is also directed along $\theta$ :

$$
\begin{aligned}
\mathbf{J} & =\mathrm{J}(\mathrm{r}, \mathrm{z}) \mathbf{u}_{\theta} \\
\mathbf{A} & =\mathrm{A}(\mathrm{r}, \mathrm{z}) \mathbf{u}_{\theta} .
\end{aligned}
$$

Under such assumptions, the problem itself can be considered axisymmetric: all the physical quantities are independent of $\theta$ and the problem is completely defined by a cross-section of the coil containing its central axis.

The reduction of the dimension of the problem has to be accounted for properly in the J-formulation by adapting the 3D Green kernel introduced in equation (14) to a 2D axisymmetric geometry. Indeed, because of the integral approach, interactions between elements of the cross-section and other elements of the coil that are no longer represented have still to be taken into account in order to properly assemble the $2 \mathrm{D}$ inductive matrix $\left[\mathrm{L}_{2 \mathrm{D}}\right]$. The $2 \mathrm{D}$ axisymmetric Green kernel, $G_{2 \text { Daxi, }}$ computed for vector fields is defined by

$$
\mathrm{G}_{2 \mathrm{Daxi}}=\int_{0}^{2 \pi} \mathrm{G}_{3 \mathrm{D}} \cdot \cos (\theta) \cdot \mathrm{rd} \theta .
$$

This relation can be expressed by an analytical formula in the cylindrical coordinate frame $(\mathrm{r}, \theta, \mathrm{z})$ as follows:

$$
\mathrm{G}_{2 \mathrm{Daxi}}=\frac{\mathrm{K}}{4 \pi \mathrm{R}}\left(\left(2-\mathrm{k}^{2}\right) \cdot \mathrm{J}_{1}(\mathrm{k})-2 \mathrm{~J}_{2}(\mathrm{k})\right)
$$

where

$$
\begin{gathered}
\mathrm{K}^{2}=(\mathrm{r}+\mathrm{R})^{2}+\mathrm{h}^{2} \\
\mathrm{k}^{2}=\frac{4 \mathrm{rR}}{\mathrm{K}^{2}} .
\end{gathered}
$$




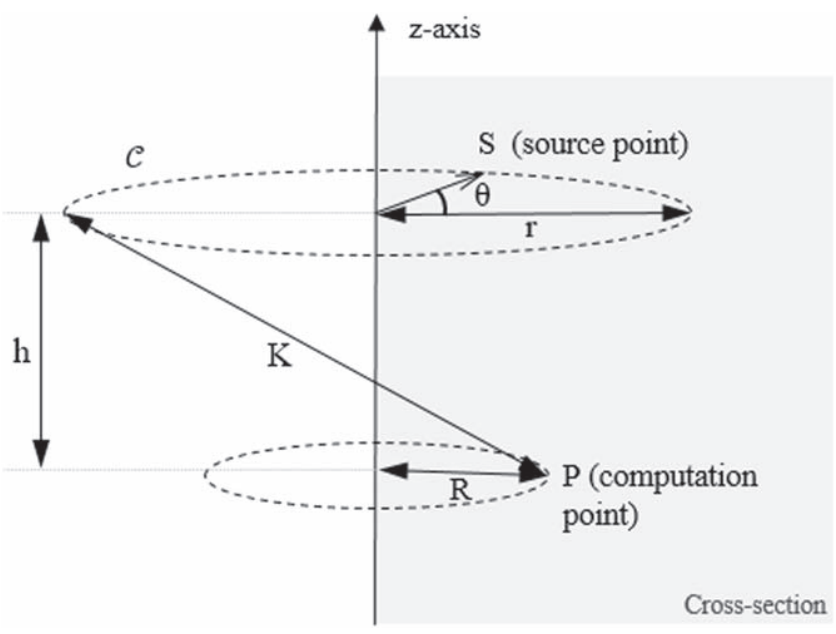

Figure 1. Integration of the 3D Green kernel (azimuthal direction).

$\mathrm{J}_{1}$ and $\mathrm{J}_{2}$ are the complete elliptic integrals of the first and second kind, respectively, and the geometrical parameters are described in figure 1.

Equations (16), (18) and (21) do not change, but the computation of the vectors and matrices is affected as follows:

$$
\begin{gathered}
\mathrm{R}_{\mathrm{ij}}=\int_{\mathrm{S}} \mathbf{w}_{\mathrm{i}} \cdot \rho(\mathrm{J}) \cdot \mathbf{w}_{\mathrm{j}} \cdot 2 \pi \mathrm{r}_{\mathrm{i}} \mathrm{dS} \\
\mathrm{L}_{2 \mathrm{Dij}}=\mu_{0} \int_{\mathrm{S}} \mathbf{w}_{\mathrm{i}} \int_{\mathrm{S}} \mathrm{G}_{2 \text { Daxi }} \cdot \mathbf{w}_{\mathrm{j}} \mathrm{d} \mathrm{S}^{\prime} 2 \pi \mathrm{r}_{\mathrm{i}} \mathrm{dS} \\
\mathrm{A}_{0 \mathrm{i}}=-\int_{\mathrm{S}} \mathbf{w}_{\mathrm{i}} \cdot \mathbf{A}_{0} \cdot 2 \pi \mathrm{r}_{\mathrm{i}} \mathrm{dS} \\
\Delta \mathrm{V}_{\mathrm{Si}}=-\int_{\mathrm{S}} \mathbf{w}_{\mathrm{i}} \cdot \Delta \mathrm{V}_{\mathrm{S}} \cdot 2 \pi \mathrm{r}_{\mathrm{i}} \mathrm{dS} .
\end{gathered}
$$

\subsection{Nonlinear behaviour}

The formulation described so far can be applied to conductive materials with any constitutive equation. In the case of superconducting materials, which exhibit a highly nonlinear voltage-current relation, several models of the constitutive law E-J have been developed, the most famous ones being the Bean model [27] and the power law model [46, 47]. For our specific problem, which is related to current distribution in a HTS coil during ramping up, the power law model (equation (32)) has been selected because it is very convenient for current variations to be considered, ranging from 0 to around $\mathrm{I}_{\mathrm{c}}$ :

$$
\rho_{\mathrm{sc}}=\frac{\mathrm{E}_{\mathrm{c}}}{\mathrm{J}_{\mathrm{c}}(\mathrm{T}, \mathbf{B})}\left(\frac{|\mathbf{J}|}{\mathrm{J}_{\mathrm{c}}(\mathrm{T}, \mathbf{B})}\right)^{\mathrm{n}(\mathrm{T}, \mathbf{B})-1} .
$$

The n-value quantifies the stiffness of the transition. The variation of $J_{c}$ with magnetic field (both magnitude and orientation) is of prime importance for coil problems [48] because each turn experiences a different local magnetic field generated by the coil itself, which may be combined with a background field (if any). The $\mathrm{J}_{\mathrm{c}}(\mathbf{B})$ curve can be determined by characterization measurements for a given temperature and under several magnetic field conditions.

To deal with E-J nonlinearity, Newton's method, which is a powerful iterative root-finding algorithm particularly adapted to stiff problems, has been implemented. The power law model, which has a continuous first-order derivative, can actually be used by such an algorithm. We used a backward Euler differentiation method for time discretization so as to ensure stability whatever the time step value is (this is particularly convenient in the case of the auto-adaptive time stepping method). The overall system to be iteratively solved is

$$
\frac{\partial \operatorname{Res}^{k}}{\partial \mathrm{I}_{L}} \cdot\left(\begin{array}{c}
\Delta \mathrm{I}_{\mathrm{L}} \\
\Delta \mathrm{V}_{\mathrm{S}}
\end{array}\right)=-\operatorname{Res}^{k}
$$

where (for a current-driven application)

$$
\begin{array}{r}
\operatorname{Res}^{\mathrm{k}}=\left(\begin{array}{cc}
{\left[\mathrm{M}_{\mathrm{RL}}\right]\left([\mathrm{R}]+\frac{1}{\Delta \mathrm{t}}[\mathrm{L}]\right)\left[\mathrm{M}_{\mathrm{RL}}\right]^{\mathrm{t}}} & {\left[\mathrm{M}_{\mathrm{s}}\right]} \\
{\left[\mathrm{M}_{\mathrm{s}}\right]^{\mathrm{t}}} & {[0]}
\end{array}\right) \\
\cdot\left(\begin{array}{c}
{\left[\mathrm{I}_{\mathrm{L}}\right]^{\mathrm{k}}} \\
{\left[\Delta \mathrm{V}_{\mathrm{S} 0}\right]^{\mathrm{k}}}
\end{array}\right)-\left(\begin{array}{cc}
{\left[\mathrm{M}_{\mathrm{RL}}\right]\left(\frac{1}{\Delta \mathrm{t}}[\mathrm{L}]\right)\left[\mathrm{M}_{\mathrm{RL}}\right]^{\mathrm{t}}} & {[0]} \\
{[0]} & {[\mathrm{Id}]}
\end{array}\right) \\
\cdot\left(\begin{array}{c}
{\left[\mathrm{I}_{\mathrm{L}}\right]^{\mathrm{k}}} \\
{\left[\mathrm{I}_{0}\right]^{\mathrm{p}+1}}
\end{array}\right)-\left(\begin{array}{c}
{\left[\mathrm{M}_{\mathrm{RL}}\right] \frac{1}{\Delta \mathrm{t}}\left(\left[\mathrm{A}_{0}\right]^{\mathrm{p}+1}-\left[\mathrm{A}_{0}\right]^{\mathrm{p}}\right)} \\
{[0]}
\end{array}\right)
\end{array}
$$

and

$$
\begin{aligned}
\frac{\partial \operatorname{Res}^{k}}{\partial \mathrm{I}_{\mathrm{L}}} & =\left(\begin{array}{cc}
{\left[\mathrm{M}_{\mathrm{RL}}\right]\left([\mathrm{R}]+\frac{1}{\Delta \mathrm{t}}[\mathrm{L}]\right)\left[\mathrm{M}_{\mathrm{RL}}\right]^{\mathrm{t}}} & {\left[\mathrm{M}_{\mathrm{s}}\right]} \\
{\left[\mathrm{M}_{\mathrm{S}}\right]^{\mathrm{t}}} & {[0]}
\end{array}\right) \\
& +\left(\begin{array}{cc}
{\left[\mathrm{M}_{\mathrm{RL}}\right][\mathrm{J}]^{\mathrm{k}}\left(\frac{\partial[\mathrm{R}]}{\partial\left[\mathrm{J}^{\mathrm{t}}\right]}\right)\left[\mathrm{M}_{\mathrm{RL}}\right]^{\mathrm{t}}} & {[0]} \\
{[0]} & {[0]}
\end{array}\right) .
\end{aligned}
$$

The index $\mathrm{p}$ refers to time domain iterations while the index $\mathrm{k}$ refers to nonlinear iterations.

\section{Model validation}

The formulation has been implemented in a multi-level and multi-method platform dedicated to low and average frequency computational magnetism. This platform is called Mipse [49] and was developed by the Grenoble Electrical Engineering Laboratory (G2ELab). It uses the Java language and offers many numerical tools particularly adapted to integral methods. 

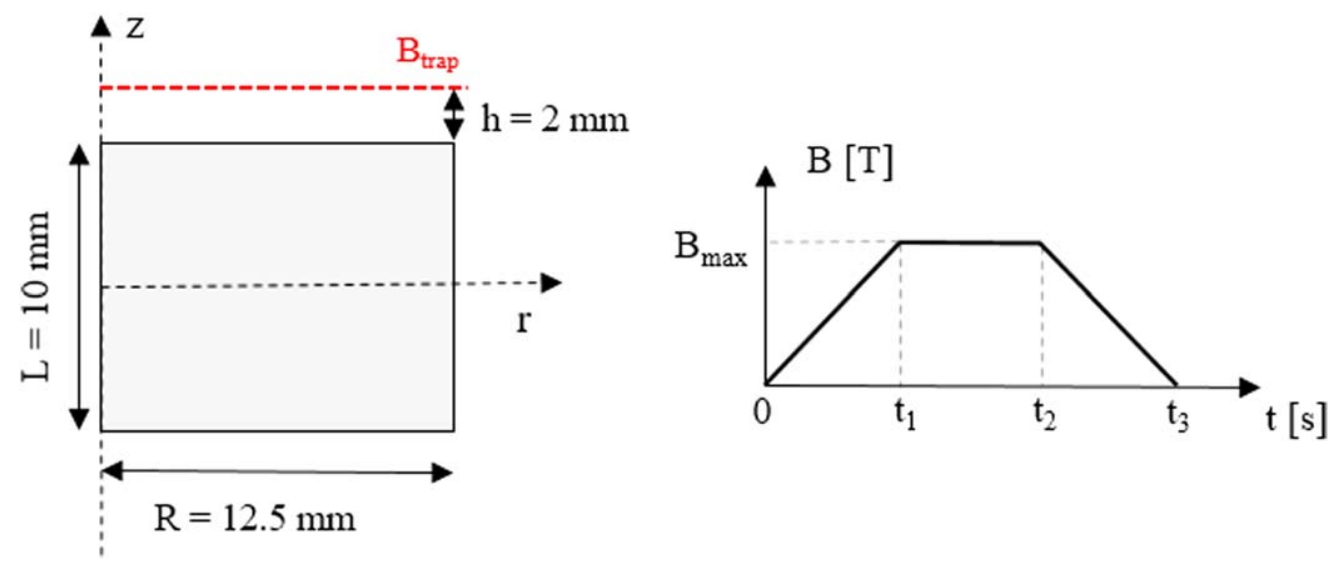

Figure 2. Bulk geometry (left) and magnetic field profile (right).

In order to validate the $2 \mathrm{D}$ axisymmetric J-formulation, a HTS bulk magnetization problem has been chosen to compare our results with a trustworthy solution obtained thanks to the $\mathrm{H}$-formulation. The problem is defined in benchmark \#4 and proposed by the HTS Modelling Workgroup [50] (results are available online).

\subsection{Test case description}

Benchmark \#4 deals with a HTS bulk cylinder subjected to zero field cooling (ZFC) magnetization, meaning that the bulk is cooled to its particular operating temperature (below its critical temperature $T_{c}$ ) before any magnetic field is applied. The bulk is $10 \mathrm{~mm}$ high and its radius is $12.5 \mathrm{~mm}$. Geometric considerations enable us to use an axisymmetric model. A uniform external magnetic field is applied along the z-direction, perpendicular to the top-bottom surface of the cylinder. The geometric specifications are displayed in figure 2, as well as the magnetic field ramp profile (with $\mathrm{B}_{\max }=1 \mathrm{~T}$ and $\left.\left[\mathrm{t}_{1} ; \mathrm{t}_{2} ; \mathrm{t}_{3}\right]=[5 ; 10 ; 15] \mathrm{s}\right)$. The critical current density is set to be homogeneous over the domain: $\mathrm{J}_{\mathrm{c}}=$ $3 \times 10^{8} \mathrm{~A} \mathrm{~m}^{-2}$. The E-J curve is modelled by a power law relation with an $\mathrm{n}$-value equal to 20 .

The problem was solved using the commercial finite element software Comsol Multiphysics. The bulk region is meshed using a mapped mesh of 800 elements (40 across, 20 down) while the air region has 1723 triangular elements.

\subsection{Bulk magnetization modelling using the J-formulation}

Although the presentation of the formulation in sections 2 and 3 was mainly focused on the current distribution problem inside HTS coils, it can of course handle other 2D axisymmetric problems such as cylinder bulk magnetization with just minor considerations.

From the point of view of an electrical circuit, the bulk can be seen as a voltage-driven device with a null voltage constraint: $\Delta \mathrm{V}_{\mathrm{s}}=0 \mathrm{~V}$. In other words, the electrical equivalent circuit generated based on the $2 \mathrm{D}$ physical domain has to be short-circuited in order to allow the current density to flow along the $\theta$-direction. In that specific case (no current generator and the magnetic field source represented by an electromotive force), equation (18) is to be solved with nonlinear terms using equation (33), where

$$
\begin{aligned}
& \operatorname{Res}^{\mathrm{k}}=\left[\mathrm{M}_{\mathrm{RL}}\right]\left([\mathrm{R}]+\frac{1}{\Delta \mathrm{t}}[\mathrm{L}]\right)\left[\mathrm{M}_{\mathrm{RL}}\right]^{\mathrm{t}}\left[\mathrm{I}_{\mathrm{L}}\right]^{\mathrm{k}} \\
& -\left[\mathrm{M}_{\mathrm{RL}}\right]\left(\frac{1}{\Delta \mathrm{t}}[\mathrm{L}]\right)\left[\mathrm{M}_{\mathrm{RL}}\right]^{\mathrm{t}}\left[\mathrm{I}_{\mathrm{L}}\right]^{p} \\
& -\left[\mathrm{M}_{\mathrm{RL}}\right] \frac{1}{\Delta \mathrm{t}}\left(\left[\mathrm{A}_{0}\right]^{p+1}-\left[\mathrm{A}_{0}\right]^{p}\right) \\
& \frac{\partial \operatorname{Res}^{\mathrm{k}}}{\partial \mathrm{I}_{\mathrm{L}}}=\left[\mathrm{M}_{\mathrm{RL}}\right]\left([\mathrm{R}]+\frac{1}{\Delta \mathrm{t}}[\mathrm{L}]\right)\left[\mathrm{M}_{\mathrm{RL}}\right]^{\mathrm{t}} \\
& +\left[\mathrm{M}_{\mathrm{RL}}\right]\left(\frac{\partial[\mathrm{R}]}{\partial\left[\mathrm{I}_{\mathrm{B}}\right]}\right)\left[\mathrm{M}_{\mathrm{RL}}\right]^{\mathrm{t}}\left[\mathrm{I}_{\mathrm{L}}\right]^{\mathrm{k}} .
\end{aligned}
$$

\subsection{Results}

With regard to mesh considerations we kept the same mapped mesh as that defined in Comsol® for the bulk region, except that no air region has to be defined in the J-formulation model. In Comsol $₫$, the problem was solved using an H-formulation with second-order elements (15 $102 \mathrm{DoF})$. The $\mathrm{J}$-formulation resolution uses a piecewise constant approximation (often used for simple mapped meshes [51, 52]) which results in 799 DoF.

Figure 3 displays the normalized superconducting loop currents induced by the uniform external magnetic field: (1) once the external field has reached $1 \mathrm{~T}\left(\mathrm{t}_{1}=5 \mathrm{~s}\right)$, (2) after a relaxation of $5 \mathrm{~s}$ still with a background field of $1 \mathrm{~T}$ $\left(t_{2}=10 \mathrm{~s}\right)$ and (3) at the end of the magnetization process, i.e. when the external field has been completely removed $\left(\mathrm{t}_{3}=15 \mathrm{~s}\right)$.

The magnetic field produced by the current distribution can be derived at any point using the Biot-Savart law. The total magnetic field (i.e. the sum of the external induction and that induced by the bulk's superconducting currents) map is computed over the bulk region at the same moments, that is to say $t_{1}, t_{2}$ and $t_{3}$ (see figure 4 ).

The trapped magnetic field profile along the radial direction, $\mathrm{B}_{\text {trap }}$, is computed $2 \mathrm{~mm}$ above the bulk top surface. 

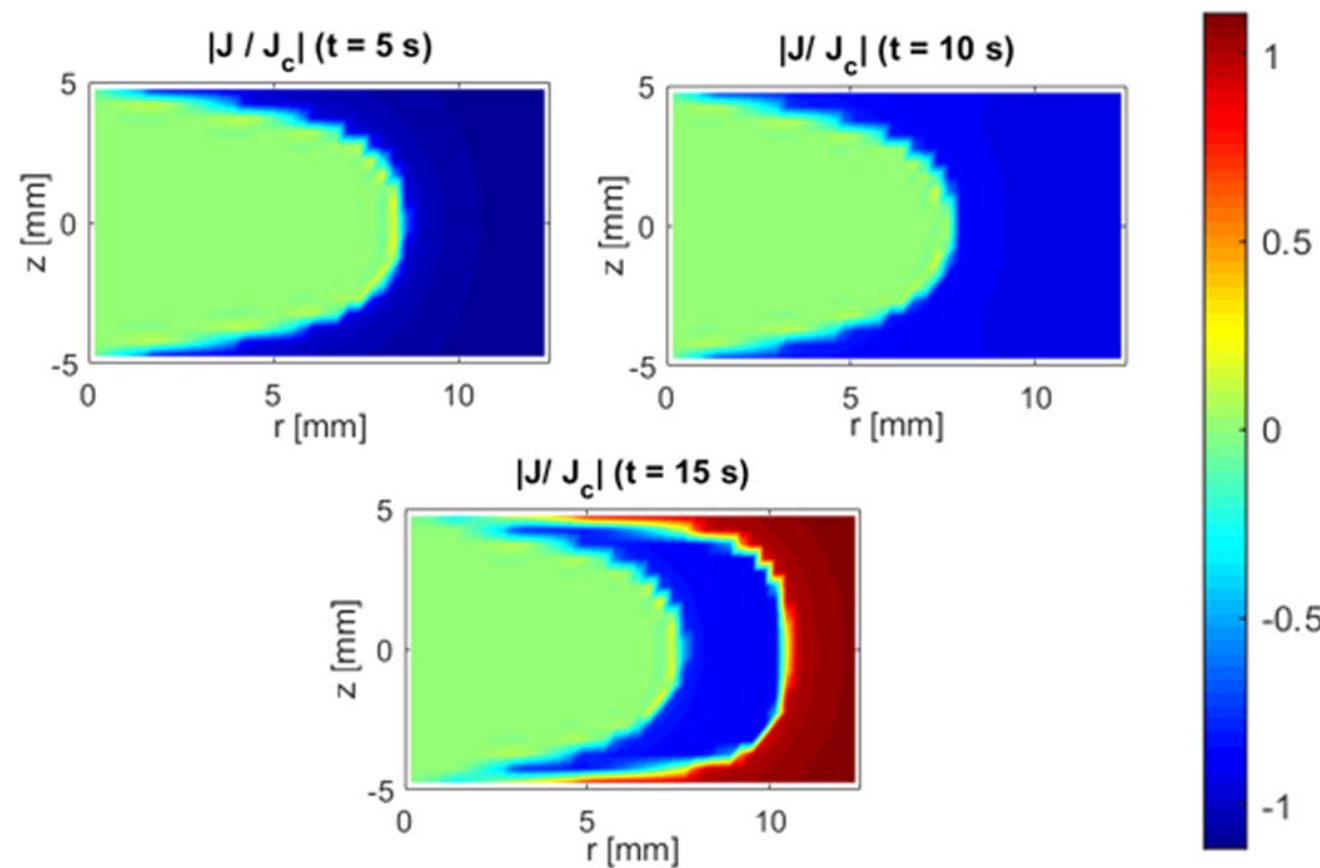

Figure 3. Normalized current distribution $\mathrm{J} / \mathrm{J}_{\mathrm{c}}$ over the $2 \mathrm{D}$ domain representing the cylinder bulk computed at $\mathrm{t}=5 \mathrm{~s}$ (top left), $\mathrm{t}=10 \mathrm{~s}$ (top right) and $\mathrm{t}=15 \mathrm{~s}$ (bottom).

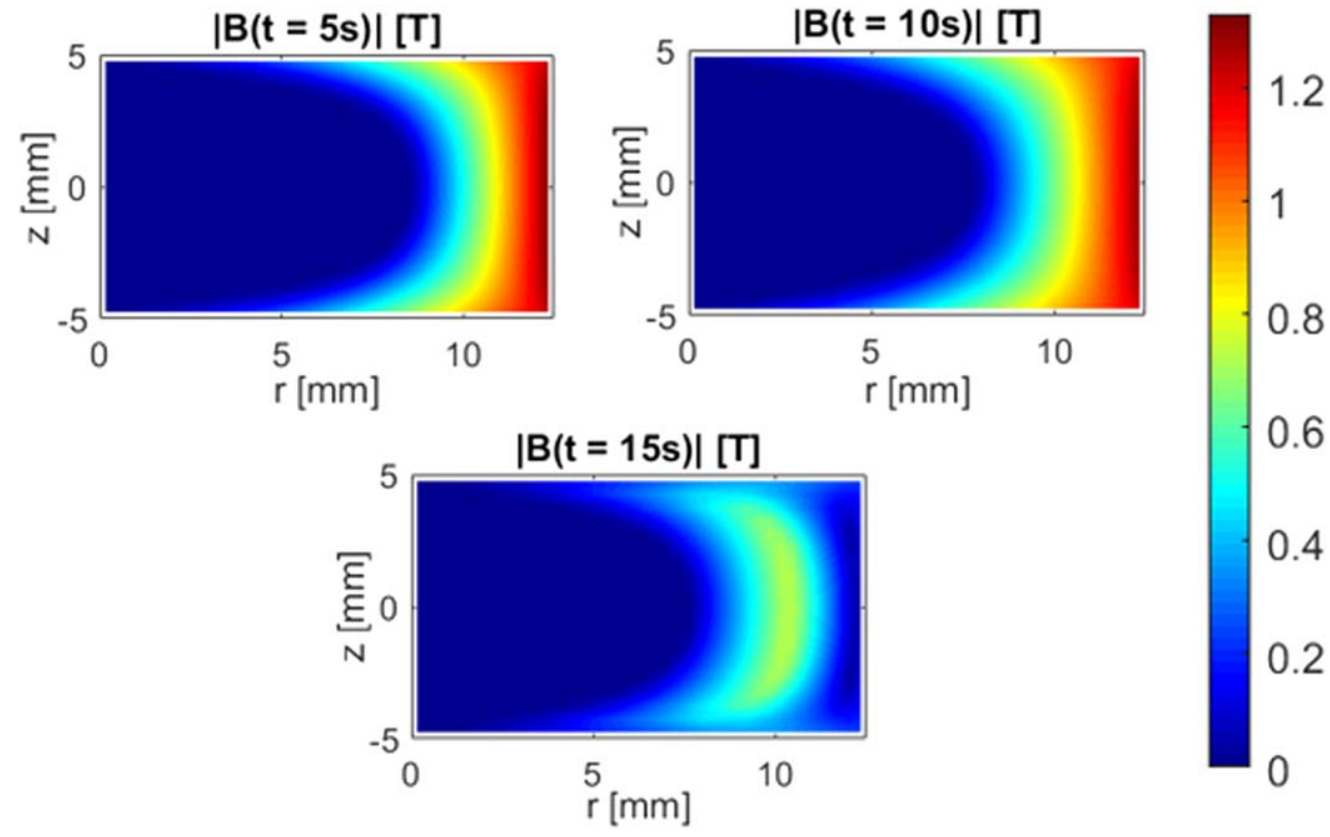

Figure 4. Induction $B$ over the $2 \mathrm{D}$ domain representing the cylinder bulk computed at $t=5 \mathrm{~s}$ (top left), $\mathrm{t}=10 \mathrm{~s}$ (top right) and $\mathrm{t}=15 \mathrm{~s}$ (bottom).

Comsol and Mipse solutions are compared in figure 5. It can be seen that the results are in quite good agreement, the maximum difference between the two codes being less than $1.12 \%$, which validates the formulation implemented in Mipse.

As expected when using a ZFC magnetization technique, $B_{\text {trap }}(r=0 \mathrm{~mm}, \mathrm{z}=7 \mathrm{~mm})$ is much lower (around $76 \mathrm{mT}$ ), once the external field has been removed, than the maximum induction value applied $\left(\mathrm{B}_{\max }=1 \mathrm{~T}\right)$. The fluxtrapping ability of the bulk is analytically estimated [53] to be $\mathrm{B}_{\text {trap,th }}(\mathrm{r}=0 \mathrm{~mm}, \mathrm{z}=7 \mathrm{~mm}) \approx 1.1 \mathrm{~T}$. This theoretical value assumes that the magnetization process has induced a homogeneous current over the cross-section which flows at its critical value; this is absolutely not the case with the magnetization scenario represented here, as shown in figure 3 .

The formulation has thus been successfully validated by comparison with a reference benchmark solution, although a more detailed comparison between both methods in terms of performance and accuracy would be interesting. 


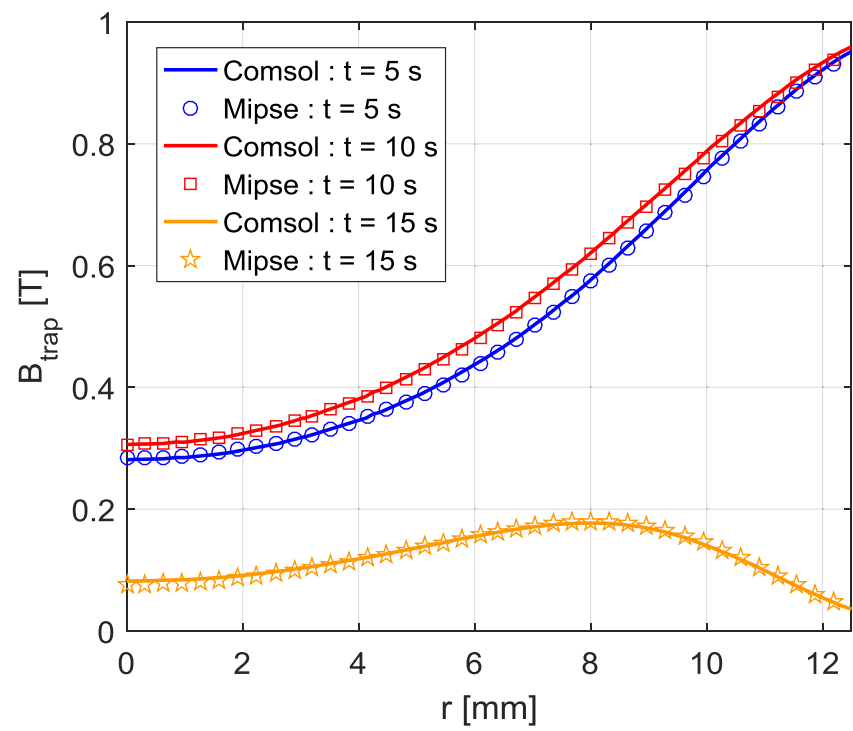

Figure 5. Trapped magnetic field along the radius computed $2 \mathrm{~mm}$ above the top surface of the bulk $(\mathrm{z}=7 \mathrm{~mm})$. Comparison of the J-formulation results (implemented in the Mipse platform) with the solution of benchmark \#4 (solid lines) solved in Comsol Multiphysics using the $\mathrm{H}$-formulation.

\section{Sensitivity analysis}

Once validated by comparison with a reference benchmark solution, the model is ready to be used for the current distribution computation inside a HTS insulated coil. Prior to the study, a characterization of the HTS tape is needed to set proper parameters of the tape's E-J curve, which in our case is modelled by a power law. The values of $n$ and $J_{c}$ can be estimated experimentally, but the accuracy depends greatly on the characterization method and the level of accuracy of the measurement devices, and can also vary from sample to sample. Thus, a sensitivity analysis has been carried out in order to investigate the influence of each parameter on the current distribution and the resulting voltage.

\subsection{Test case presentation}

For reasons of computation time, we defined a small-scale single pancake coil made of 25 turns with a deliberately small inner radius of $5 \mathrm{~mm}$ in order to generate a magnetic field that is not too small despite the reduced number of turns. For such geometry, the axisymmetric condition is satisfied.

The tape is $12 \mathrm{~mm}$ wide and the superconducting layer is $2 \mu \mathrm{m}$ thick (the total thickness of the tape is $139.5 \mu \mathrm{m}$ plus $20 \mu \mathrm{m}$ thick insulating layers on the top and bottom).

In this approach, no current sharing between the conducting layers of the tape is considered, otherwise thermal coupling should be added to account for the proper current distribution among layers. Therefore, current is assumed to flow only through the superconducting layer. This assumption is correct as long as the critical current value is not exceeded. As the superconducting layer is the only active one there is no need to mesh others. Furthermore, due to the very high aspect

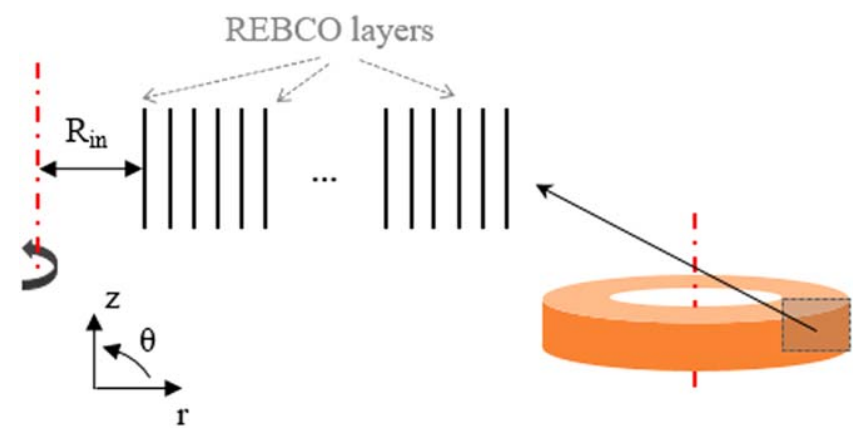

Figure 6. Simplified coil geometry represented in a 2D axisymmetric view.

ratio (6000) between the thickness and width of the superconducting layer, a thin regions approximation is used to represent the tape. Therefore, proximity effects are neglected (the distance between turns being around 90 times larger than the represented turn's thickness) and the current density is assumed to be uniform over the superconducting layer thickness. The mesh is thus considerably reduced: each turn is represented by a line in the 2D domain which is divided in that case into 100 elements. The geometry is displayed in figure 6.

\section{2. $n$-value and $J_{C}(B, \theta)$ variations}

In this section, the impact of the n-value parameter as well as variations in $\mathrm{J}_{\mathrm{c}}$ are investigated over the coil's voltage, which is the quantity of interest for practical measurements with regard to the quench detection. A constant n-value, $\mathrm{n}(\mathrm{T}, \mathbf{B})=\mathrm{n}$, is assumed here.

The gradual penetration of current from the edges to the centre of each turn creates an inductance variation and a voltage increase is observed even for a constant current ramp rate. In order to quantify the impact of both parameters on the current distribution, we chose to compare the interpolated voltage slope (the voltage computed corresponds to the total voltage of the coil) due to current penetration for several $n$-values and $\mathrm{J}_{\mathrm{c}}(\mathrm{B}, \theta)$ characteristics for the same current ramp rate of $1 \mathrm{As}^{-1}$.

5.2.1. $n$-value sensitivity. In the first scenario, the $n$-value of the power law varies between 20 and 50, which is a typical range of variation for HTS materials [54], while the $\mathrm{J}_{\mathrm{c}}(\mathrm{B}, \theta)$ curve does not change and is set to a reference curve, $\mathrm{J}_{\mathrm{c}}(\mathrm{B}, \theta)_{\text {ref }}$, corresponding to data reported in [55]. V-I curves obtained for several $n$-values are displayed in figure 7 . The ripples observed are due to the piecewise constant approximation and have of course no physical meaning. Indeed, the current starts to flow very close to the edges and gradually penetrates towards the centre: for a given turn, once the current density of an element gets closer to its critical value, current begins to flow through the next element, explaining the staircase-like appearance of the V-I curve. It 


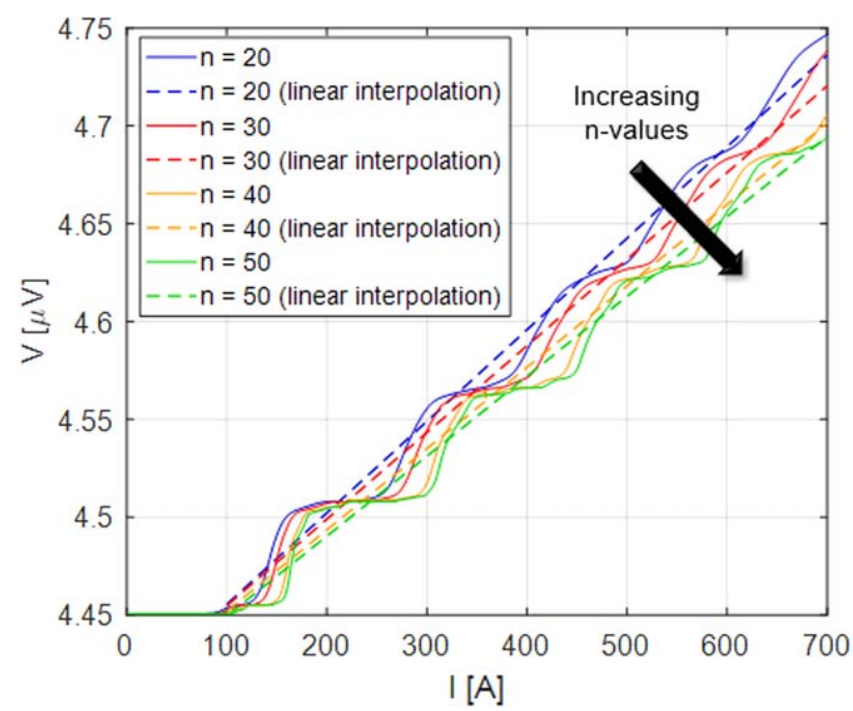

Figure 7. V-I curves computed for several n-values.

tends to become smoother with an increased number of elements and the gradual penetration of the current.

5.2.2. $J_{C}(T, B, \theta)$ sensitivity. The critical current depends on the temperature and the magnetic field (magnitude and angle) experienced locally by the tape, as well as the tape's local performance. That performance is usually estimated by the supplier in terms of local critical current at $77 \mathrm{~K}$ and under self-field $I_{c \text { s.f. } 77 \mathrm{~K}}$, obtained by continuous contactless measurement [56].

For a given sample, we can thus define the lift factor LF as the ratio between the critical current $\mathrm{I}_{\mathrm{c}}\left(\mathrm{T}_{\mathrm{op}}, \mathrm{B}, \theta\right)$ measured at a given operating temperature in a given magnetic field and its value at $77 \mathrm{~K}$ self-field:

$$
\begin{aligned}
\mathrm{I}_{\mathrm{c}}\left(\mathrm{T}_{\mathrm{op}}, \mathrm{B}, \theta\right)_{\text {tape }} & =\frac{\mathrm{I}_{\mathrm{c}}\left(\mathrm{T}_{\mathrm{op}}, \mathrm{B}, \theta\right)_{\text {sample }}}{\mathrm{I}_{\mathrm{c}}(77 \mathrm{~K}, \mathrm{sf})_{\text {sample }}} I_{\mathrm{c}}(77 \mathrm{~K}, \mathrm{sf})_{\text {tape }} \\
& =\mathrm{LF}\left(\mathrm{T}_{\mathrm{op}}, \mathrm{B}, \theta\right) I_{\mathrm{c}}(77 \mathrm{~K}, \mathrm{sf})_{\text {tape }} .
\end{aligned}
$$

The lift factor is very dependent on the manufacturing process, but for practicality we will first assume that it remains identical for lengths of tape produced by the same supplier production line at roughly the same period.

Characterization measurements to obtain $\mathrm{I}_{\mathrm{c}}(4.2 \mathrm{~K}, \mathrm{~B}, \theta)$ were previously carried out on SuperOx samples [55]. We then added the variation with temperature by using an analytical formula derived in [57] from experiments on similar tapes from SuperOx, in order to obtain a reference lift factor $\mathrm{LF}_{\text {ref }}(\mathrm{T}, \mathrm{B}, \theta)$.

In order to study the impact of the variation and/or uncertainty on the input data for the lift factor value, a second sensitivity analysis was carried out, this time keeping the n-value constant at 25 . We let the lift factor vary from 0.5 to 1.2 times the reference value. Figure 8 shows the V-I curves obtained depending on the LF parameter: the interpolated slope decreases significantly as the performance increases (higher LF). Indeed, the penetration speed of the current

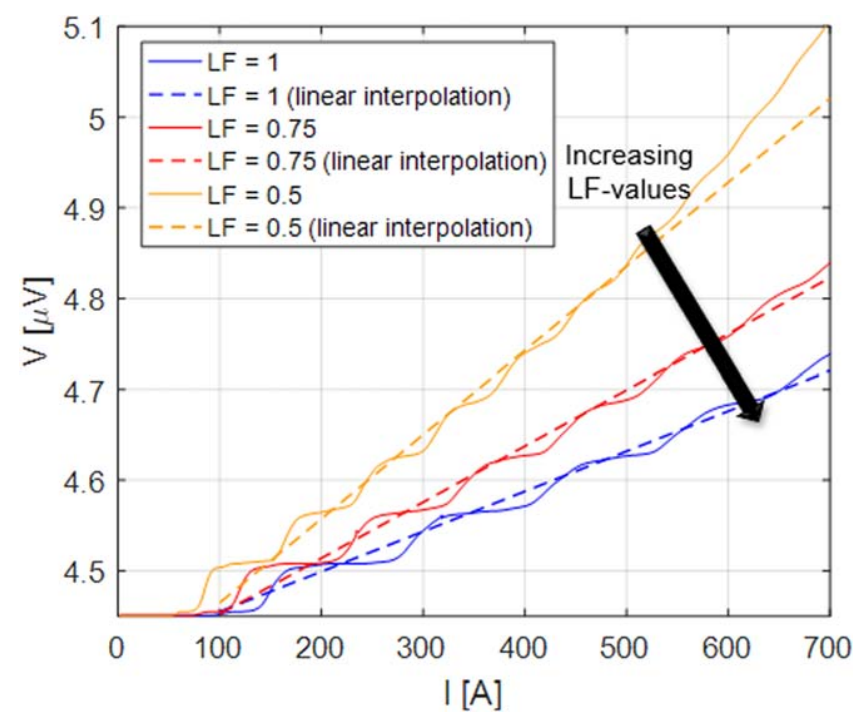

Figure 8. V-I curves computed for $\mathrm{n}=25$ and several $\alpha$-values.
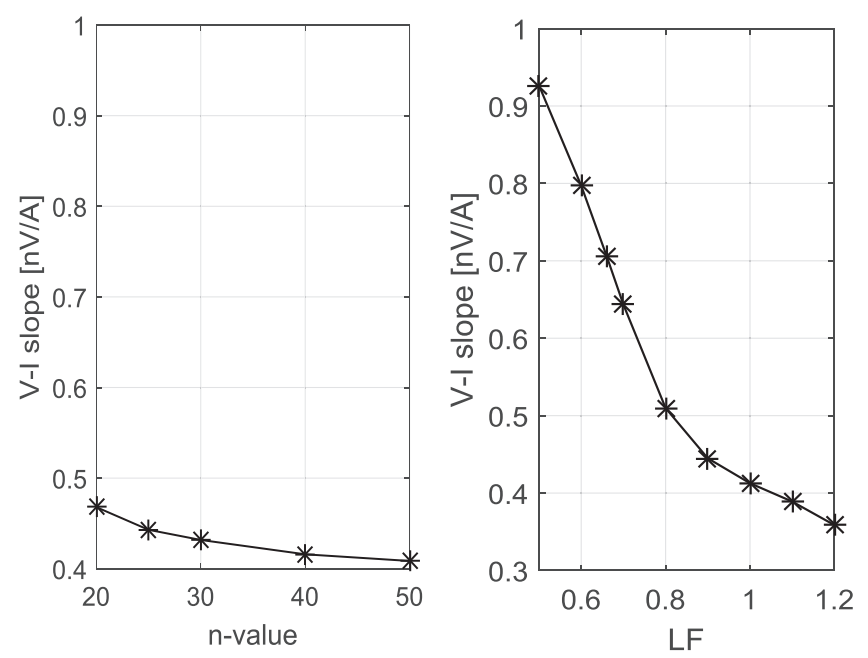

Figure 9. Sensitivity of the model to the n-value (left) and the $\mathrm{J}_{\mathrm{c}}(\mathrm{B}, \theta)$ curve (right) - the same scale on y-axis is used for both plots.

density from the tape edges to the centre is higher at lower $\mathbf{J}_{\mathrm{c}}$ as the edges become 'saturated' for smaller current densities.

5.2.3. Sensitivity analysis: conclusions. Figure 9 displays an overview of the sensitivity analysis based on its impact on the V-I interpolated slope. It can be concluded that the model is quite robust regarding variations in the $n$-value. The equivalent $\mathrm{V}-\mathrm{I}$ slope depends much more on fluctuations in the lift factor, which makes it difficult to predict the behaviour of a given coil as lift factor values are not usually known with a good level of confidence. For instance, if the tape performances are overestimated in the model, the simulated V-I curve will increase more slowly compared with experimental data.

However, that high sensitivity may have an unexpected positive consequence: the comparison between the voltage variation during the ramp-up of an actual coil and the expected values derived from simulation could be a good way to evaluate the practical $\mathrm{I}_{\mathrm{c}}$ of that given coil without having to 
get sufficiently close to it for dissipation to appear, thus enabling a safe check of operational limits (see section 6.3).

\section{Comparison with experimental data}

\subsection{Experimental case and simulation setup}

A double pancake coil made of $12 \mathrm{~mm}$ insulated REBCO tape from SuperOx has been tested in self-field conditions. The average critical current of the tape was evaluated based on SuperOx data from Tapestar characterization (77 K, self-field) and was found to be $370 \mathrm{~A}$. Its inner radius is $96 \mathrm{~mm}$, the top pancake has 150 turns and the bottom pancake has 168 turns. The superconducting layer is $2 \mu \mathrm{m}$ thick for a total tape thickness of $139 \mu \mathrm{m}$ (without insulation). Once cooled down to $4.2 \mathrm{~K}$ (in a liquid helium bath), the coil is ramped up at a rate of $1 \mathrm{~A} \mathrm{~s}^{-1}$. The objective is to compare simulation results obtained with the model presented in this paper with experimental data on the current distribution in HTS REBCO coils.

The model geometry is built based on a thin-strip approximation applied to the superconducting layer (as in section 5). Each turn is divided into 50 elements of the same width, leading to a final mesh made of 15900 elements.

Regarding the tape properties, the n-value of the power law is set to 25. Variation of $\mathrm{J}_{\mathrm{c}}$ with the magnetic field is defined using the lift factor derived from SuperOx data and introduced previously in the sensitivity analysis (38), with $\mathrm{I}_{\mathrm{c}}\left(\mathrm{T}_{\mathrm{op}}, \mathrm{B}, \theta\right)_{\text {sample }}$ the critical current map measured in [55] for several operating conditions on a $4 \mathrm{~mm}$ sample, $\mathrm{I}_{\mathrm{c}}(77 \mathrm{~K}, \mathrm{sf})_{\text {sample }}=135 \mathrm{~A}$ is its critical current at $77 \mathrm{~K}$, selffield. $I_{c}(77 \mathrm{~K}, \mathrm{sf})_{\text {tape }}=370 \mathrm{~A}$ is the critical current of the $12 \mathrm{~mm}$ tape used for the double pancake coil.

The simulation is run at the same speed as for the real experiment, with a $1 \mathrm{~A} \mathrm{~s}^{-1}$ current ramp, starting from a homogeneous initial current density of zero. Because this problem deals with full matrices whose size is quite important (15 583 by 15583 ) and the code has not been optimized for fast computation purposes it took several days to reach few hundred amperes on a desktop computer. This is the reason why the computation was stopped at only $350 \mathrm{~A}$ in both cases, although experimental data are available for higher current values.

\subsection{Comparison results: influence of the lift factor}

Figure 10 displays the experimental values of total coil voltage (grey curve, solid line) along with the simulation results based on tape characterization (simulation 1, black curve, broken line). The simulated coil voltage shows a trend similar to the experimental data, with an identical starting point and quasi-linear rise.

However, the slope of the voltage rise is underestimated by $36.3 \%$ compared with the voltage measurements. This might be explained by an overestimation of the lift factor of the tape. As mentioned in section 5.2.2, we assumed that the lift factor remains constant for a given manufacturer's production. This is an over-simplification, even in the case of

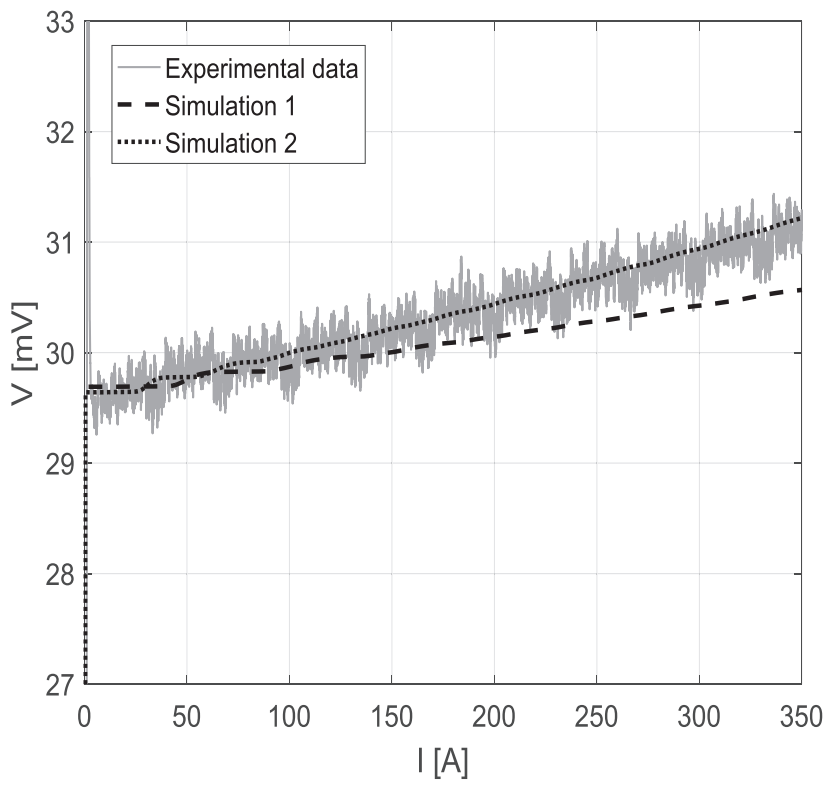

Figure 10. Voltage-current characteristic of a double pancake coil ramped up at $1 \mathrm{~A} \mathrm{~s}^{-1}$. Comparison between experimental data (solid line) and simulation results (broken and dotted lines).
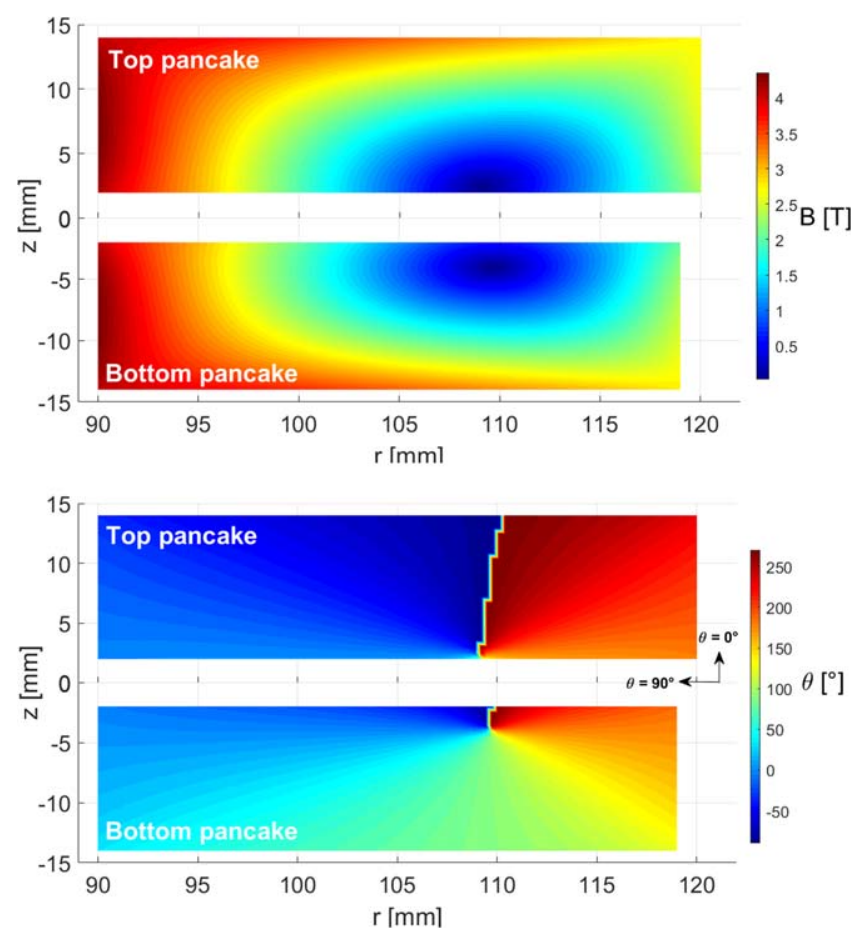

Figure 11. Magnetic field distribution (amplitude and incident angle) inside the double pancake computed for an input current of $795 \mathrm{~A}$ (magnetostatic study).

REBCO tapes without artificial pinning. Accumulated data from SuperOx samples characterized by different laboratories around the world show that the evaluated lift factor may have a non-negligible spread ( $\pm 30 \%$ at most) [58]. Another reason for that discrepancy may be that the operating temperature during the experiment was not exactly $4.2 \mathrm{~K}$. An operating temperature of $5 \mathrm{~K}$ instead of $4.2 \mathrm{~K}$ leads to a $5 \%$ reduction in $\mathrm{I}_{\mathrm{c}}$. Such a slight increase in temperature could happen locally 

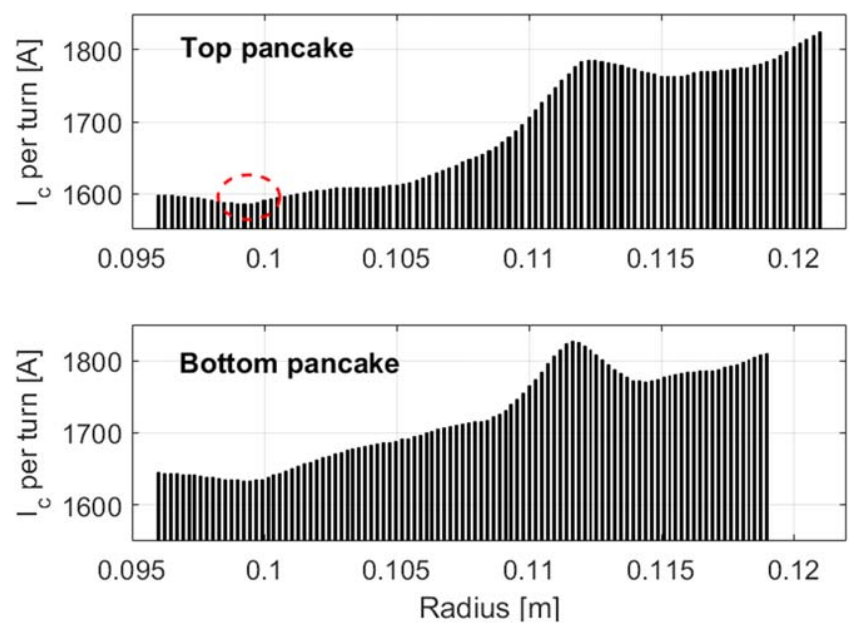

Figure 12. Estimated critical current per turn $I_{c}{ }^{\text {turn }}$ with the initial $\mathrm{J}_{\mathrm{c}}(\mathrm{T}, \mathrm{B}, \theta)$ curve. Top and bottom pancakes results are displayed in the top and bottom plots, respectively. The dotted red line shows the smallest value obtained at turn number 14 (starting from the innermost turn).

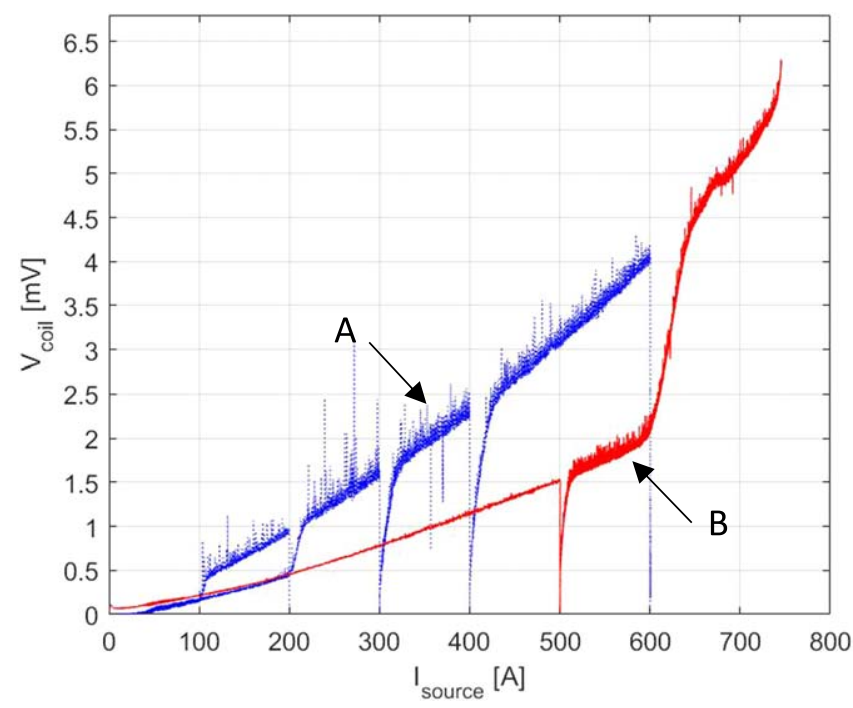

Figure 13. Magnetization effect observed on a double pancake coil. The blue curve (A) corresponds to the first current ramp $\left(1 \mathrm{~A} \mathrm{~s}^{-1}\right.$ until $600 \mathrm{~A}$ ) and the red curve (B) corresponds to a second ramping up at the same rate but to a higher current value.

at the junction of the current leads or in the middle of the coil where there is less exchange with the cooling bath due to the mechanical structure.

Considering all the possible errors just mentioned, simulations have been launched with reduced lift factors. A good agreement is obtained with a lift factor reduced by $28 \%$, as shown in figure 10 by the V-I curve labelled 'Simulation 2' (black, dotted line).

\subsection{Discussion}

Firstly, this study demonstrates that the model is able to simulate a full-sized 318-turn double pancake coil made of more than $300 \mathrm{~m}$ of tape. The model provides an explanation
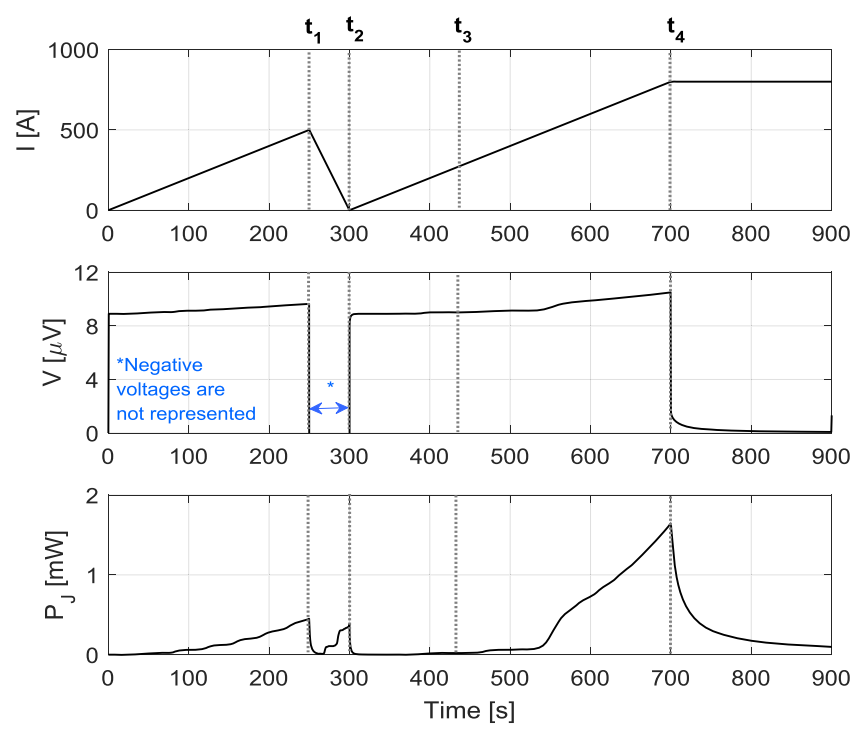

Figure 14. Current generator (top), total coil voltage (middle) and Joule losses (bottom) versus time.

for the quasi-linear voltage rise observed experimentally during ramp-up. Quantitatively, the results are not in perfect agreement if we rely on tape characterization from the literature, but that was to be expected considering the spread observed in such measurements. Perfect agreement, however, is obtained for lift factor values that are plausible, which give us confidence in the validity of the model.

As was mentioned in section 5.2.3, high sensitivity of the simulation results to the input critical current performances could be an effective way of evaluating the critical current without risk. In the case of the studied pancake coil, a rough estimation of that critical current was made by a magnetostatic study with the hypothesis of homogeneous current distribution to get the local magnetic field amplitude and orientation (see figure 11), and then evaluating the critical current based on the expected lift factor, as shown in figure 12. It has to be noticed that both single pancake coils (top and bottom ones) do not have exactly the same outer radius or the same number of turns due to practical winding constraints. This introduces an asymmetry of the magnetic flux distribution with respect to the medium plane and thus results in a slight difference in the $I_{c}$ value per turn for each coil. Using that technique the coil's limiting critical current was estimated to be around $1545 \mathrm{~A}$.

Assuming a $28 \%$ reduction in the lift factor, the revised critical current would be around $1112 \mathrm{~A}$. The next step will be to go up to that value experimentally to corroborate this new estimate.

Finally, this pancake was made in the framework of a SMES project [17] for which several identical pancakes will be made and tested, with increasing tape performances. We intend to compare the voltage slope on each of them in order to see if a variation of the voltage rise can indeed be observed and correlated with the tape performance. 


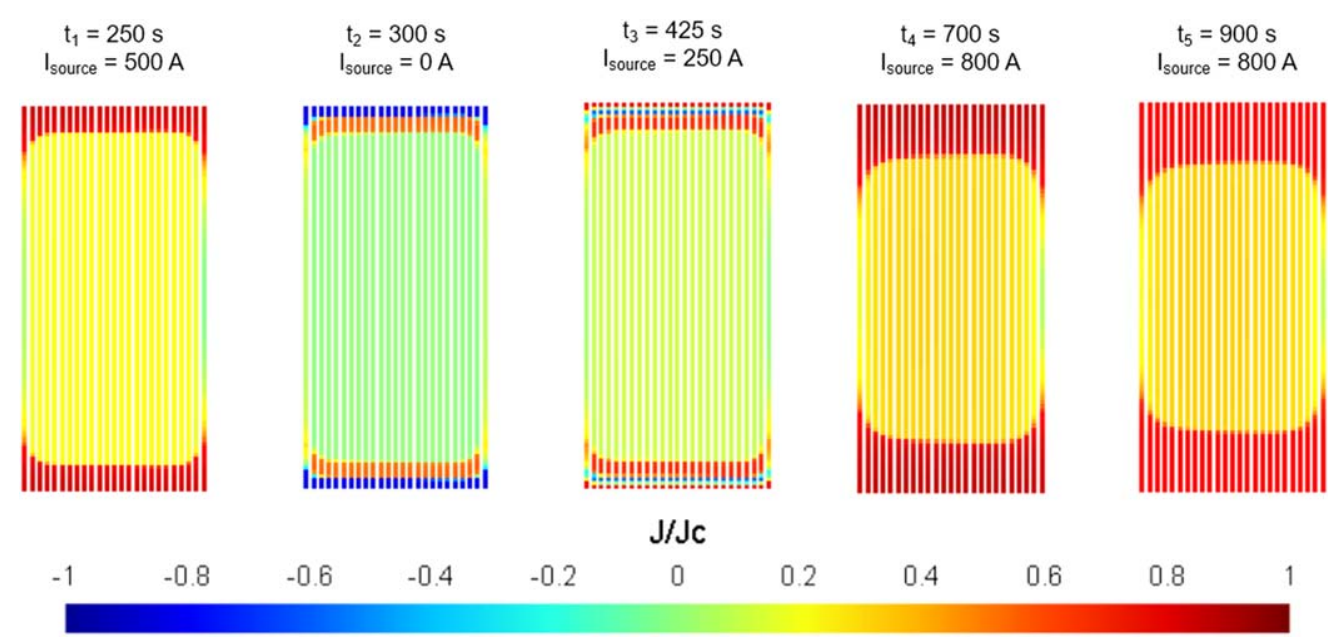

Figure 15. Normalized current density distribution along the tape width for the 25-turn coil (not to scale: the turn thickness has been enlarged for visibility reasons).

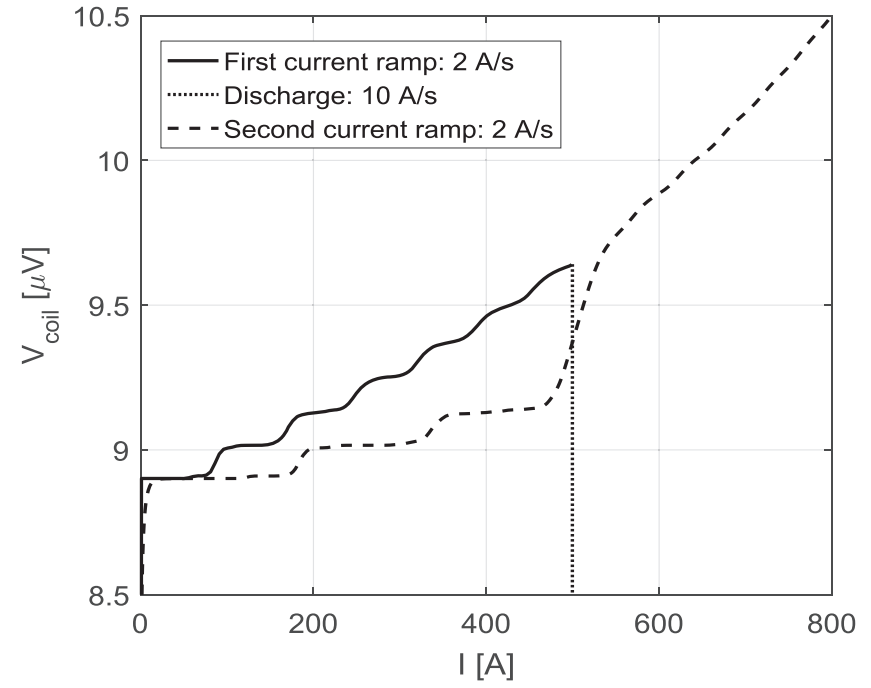

Figure 16. V-I curve computed for the 25-turn coil (with zoom on the voltage increase due to magnetization effects only).

\section{Application of model to coil current density distribution hysteresis}

In this section, the model is applied to the small-scale test case previously introduced in section 5 to study the evolution of the coil current density distribution when several charges and discharges of the coil are performed without warming up. The resulting behaviour is hysteretic and can be interpreted as 'magnetization' or 'shielding current'. Our point of view here is focused on the resulting voltage, but the same model could be used without any change to obtain the temporal variation of field homogeneity for example.

\subsection{Motivation and experimental results}

As already said in the Introduction, the concept of early detection for quench protection may be difficult to implement if transient voltages with similar dynamics and amplitude to that of a transition is observed on the coil voltage due to magnetization. Such a transient voltage depends not only on the current ramp rate as it would for normal coil, but also on the 'magnetization state' of the coil, i.e. on the previous current distributions the coil has experienced since it has been cooled down. Figure 13 displays the V-I curve obtained experimentally for the double pancake coil presented in section 6 when submitted to a first current ramp of $1 \mathrm{~A} \mathrm{~s}^{-1}$, then discharged to zero current and ramped up again at the same rate but to a higher current value than the first time.

The voltage variation observed around $600 \mathrm{~A}$ during the second ramp (red curve) could easily be misinterpreted as the onset of a transition, although it is only due to magnetization effects as the voltage increase slows down from $650 \mathrm{~A}$ until recovering the same slope as for the first ramp. Thus, such dynamic behaviour is to be estimated beforehand if one wants to be able to identify if the voltage variations observed during experiments originate from dynamic current distribution or from dissipation. This information is of prime importance to enable early detection and to automate the protection system with a criterion based on a dissipative threshold voltage value while avoiding false quench detection.

\subsection{Small-scale insulated coil}

The model can help to estimate such a transient behaviour provided that accurate data related to the tape characterization are available, as demonstrated in section 6 . In this sub-section, the small-scale test case defined by the 25-turn single pancake coil introduced in the sensitivity analysis is used to reduce the number of DoF and thus decrease significantly the computation time to enable the study of more complex scenarios than the one in section 6 . Therefore, the coil is submitted to a typical current profile that could be followed when testing a HTS magnet. Once the coil is cooled to its operating temperature of $4.2 \mathrm{~K}$, a first current ramp of $2 \mathrm{~A} \mathrm{~s}^{-1}$ is applied until $500 \mathrm{~A}$ (from 0 to $\mathrm{t}_{1}$ ), followed by a discharge to zero current $\left(t_{1}\right.$ to $\left.t_{2}\right)$. The current is increased again at a same rate until it reaches $800 \mathrm{~A}\left(t_{2}\right.$ to $\left.t_{4}\right)$, then a plateau lasting $200 \mathrm{~s}$ is simulated ( $t_{4}$ to end). The total simulation lasted for a few 
hours on a desktop computer. Figure 14 shows the time evolution of the current generator, the coil's voltage end-toend and the Joule losses inside the coil. These plots have to be connected to the normalized current distribution $\left(\mathrm{J} / \mathrm{J}_{\mathrm{c}}\right)$ displayed in figure 15 for several time values $t_{i}$ referenced in figure 14 .

The first magnetization slope appears from 0 to $t_{1}$ : it has a linear behaviour due to the gradual penetration of the current from the edges to the centre. The negative voltage due to the decrease in current between $t_{1}$ and $t_{2}$ is not displayed in order to zoom in on the voltage range of interest, i.e. positive voltages. Negative current densities penetrating the tapes are observed, resulting in a different current distribution at the end of the discharge stage at $t_{2}$ compared with the initial one, although in both cases there is no transport current. Due to the magnetization effect, the slope observed between $t_{2}$ and $t_{3}$ is almost halved compared with the one between $0 \mathrm{~s}$ and $\mathrm{t}_{1}$ even if the ramp rate is the same. Just before reaching $t_{3}$ (around $480 \mathrm{~A}$ ), the voltage increases significantly until $530 \mathrm{~A}$ where the initial slope of the V-I curve is recovered: current continues to penetrate more deeply inside each turn. Such a magnetization effect, represented in figure 16, follows closely the one observed during experiments (figure 13). It is worth noticing that almost no Joule losses are generated during such a voltage increase (see figure 14, bottom plot), which definitely differentiates it from a quench signal.

The relaxation starting at $t_{4}$ until $t_{e n d}=900 \mathrm{~s}$ is characterized by a near-zero voltage value (transport current variation no longer present). Current continues to penetrate but at a slower rate so as to tend to a homogeneous distribution after a very long time. It has to be noted that a nonzero voltage value during relaxation can be observed because of a non-zero resistance. This is a side effect of using a power law model, in which the dissipative voltage is never truly zero. The use of an alternative power law, such as the one proposed in [59], would lead to a strictly 0 value.

\section{Conclusion}

This paper deals with the current distribution problem in insulated HTS coils wound with REBCO tape, using an original approach based on a volume integral formulation. The so-called J-formulation is introduced for general 3D cases and is then detailed for the more specific case of HTS coils by adding a 2D axisymmetric condition and introducing nonlinearities. Such a choice was motivated by the specificity of the volume integral formulation to mesh only active regions, reducing the size of the problem significantly. This is particularly attractive for REBCO HTS coil geometries as the active regions are actually represented by the thin film superconducting layer only, resulting in large air gaps between two conducting regions. Moreover, the use of facet elements straightforwardly leads to circuit coupling, enforcing strictly the current conservation, which is very convenient for convergence considerations.

The model was successfully validated by firstly comparing results with a reference benchmark solution dealing with HTS bulk magnetization. It was then applied to a realscale double pancake coil for comparison with experimental data. Results showed the high sensitivity of the model to $J_{c}$ values, which could actually allow for an original way of evaluating safely the limiting $I_{c}$ of a coil without the need to ramp it all the way to the limit. Finally, the model was applied to a small-scale example so as to study the hysteretic behaviour of the current density distribution within each turn. The complex voltage variations experimentally observed have been successfully reproduced. The model is thus able to estimate the transient behaviour of the coil, which is of prime importance for designing efficient protection systems and to automate them. Model improvements are still ongoing with regard to computation times in order to be able to simulate bigger coil geometries within reasonable computation times.

\section{Acknowledgments}

The authors would like to thank Kevin Berger and Jakub Kapek from the Université de Lorraine (GREEN lab), for their help with setting up the Comsol model for first comparisons and J Ciceron, A-J Vialle and P Tixador for fruitful discussions.

\section{ORCID iDs}

Rozier Blandine (ib https://orcid.org/0000-0002-2945-8918

Badel Arnaud (10 https://orcid.org/0000-0002-5273-2612

\section{References}

[1] Godeke A et al 2007 Limits of $\mathrm{NbTi}$ and $\mathrm{Nb}_{3} \mathrm{Sn}$ and development of W\&R Bi-2212 high field accelerator magnets IEEE Trans. Appl. Supercond. 17 1149-52

[2] Miller J R and Bird M D 2004 A new series-connected hybrid magnet system for the national high magnetic field laboratory IEEE Trans. Appl. Supercond. 14 1283-6

[3] Lécrevisse T, Badel A, Benkel T, Chaud X, Fazilleau P and Tixador P 2018 Metal-as-insulation variant of no-insulation HTS winding technique: pancake tests under high background magnetic field and high current at $4.2 \mathrm{~K}$ Supercond. Sci. Technol. 31055008

[4] Markiewicz W D et al 2012 Design of a superconducting $32 \mathrm{~T}$ magnet with REBCO high field coils IEEE Trans. Appl. Supercond. 224300704

[5] Weijers H W et al 2016 Progress in the development and construction of a 32-T superconducting magnet IEEE Trans. Appl. Supercond. 264300807

[6] MagLab claims record with novel superconducting magnet https://nationalmaglab.org/news-events/news/maglabclaims-record-with-novel-superconducting-magnet (accessed 6 February 2019)

[7] Wang Q et al 2013 High magnetic field superconducting magnet system up to $25 \mathrm{~T}$ for ExCES IEEE Trans. Appl. Supercond. 234300905

[8] Liu J H et al 2015 Development of YBCO insert for a $25 \mathrm{~T}$ all superconducting magnet 2015 IEEE Int. Conf. on Applied Superconductivity and Electromagnetic Devices (ASEMD) pp 433-4 
[9] Liu J, Dai Y and Li L 2016 Progress in the development of a $25 \mathrm{~T}$ all superconducting NMR magnet Cryogenics 79 $79-84$

[10] Awaji S et al 2017 First performance test of a 25 T cryogenfree superconducting magnet Supercond. Sci. Technol. 30 065001

[11] Wang Y, Chan W K and Schwartz J 2016 Self-protection mechanisms in no-insulation ( $\mathrm{RE}) \mathrm{Ba}_{2} \mathrm{Cu}_{3} \mathrm{O}_{x}$ high temperature superconductor pancake coils Supercond. Sci. Technol. 29045007

[12] Song J-B, Hahn S, Lécrevisse T, Voccio J, Bascuñán J and Iwasa Y 2015 Over-current quench test and self-protecting behavior of a $7 \mathrm{~T} / 78 \mathrm{~mm}$ multi-width no-insulation REBCO magnet at 4.2 K Supercond. Sci. Technol. 28114001

[13] Lécrevisse T and Iwasa Y 2016 A (RE)BCO pancake winding with metal-as-insulation IEEE Trans. Appl. Supercond. 26 4700405

[14] Wang X, Trociewitz U P and Schwartz J 2007 Near-adiabatic quench experiments on short $\mathrm{YBa}_{2} \mathrm{Cu}_{3} \mathrm{O}_{7-\delta}$ coated conductors J. Appl. Phys. 101053904

[15] Kim W-S, Trillaud F, Ang I C, Hahn S and Iwasa Y 2007 Normal zone propagation in YBCO winding pack models IEEE Trans. Appl. Supercond. 17 2478-81

[16] Badel A, Tixador P, Simiand G and Exchaw O 2010 Quench detection system for twin coils HTS SMES Cryogenics $\mathbf{5 0}$ 674-81

[17] Ciceron J, Badel A, Tixador P, Pasquet R and Forest F 2018 Test in strong background field of a modular element of a REBCO 1 MJ high energy density SMES IEEE Trans. Appl. Supercond. 285701005

[18] Deng X et al 2015 An experimental and numerical study on the inductance variation of HTS magnets IEEE Trans. Appl. Supercond. 254603005

[19] Grilli F and Ashworth S P 2007 Measuring transport AC losses in YBCO-coated conductor coils Supercond. Sci. Technol. 20 794-9

[20] Pardo E 2008 Modeling of coated conductor pancake coils with a large number of turns Supercond. Sci. Technol. 21065014

[21] Prigozhin L and Sokolovsky V 2011 Computing AC losses in stacks of high-temperature superconducting tapes Supercond. Sci. Technol. 24075012

[22] Pardo E 2016 Modeling of screening currents in coated conductor magnets containing up to 40000 turns Supercond. Sci. Technol. 29085004

[23] Ueda $\mathrm{H}$ et al 2016 Numerical simulation on magnetic field generated by screening current in 10-T-class REBCO coil IEEE Trans. Appl. Supercond. 264701205

[24] Norris W T 1970 Calculation of hysteresis losses in hard superconductors carrying ac: isolated conductors and edges of thin sheets J. Phys. D: Appl. Phys. 3 489-507

[25] Brandt E H 1996 Superconductors of finite thickness in a perpendicular magnetic field: strips and slabs Phys. Rev. B 54 4246-64

[26] Carr W J 2001 AC Loss and Macroscopic Theory of Superconductors. (Boca Raton, FL: CRC Press)

[27] Bean C P 1964 Magnetization of high-field superconductors Rev. Mod. Phys. 36 31-9

[28] Grilli F, Brambilla R, Sirois F, Stenvall A and Memiaghe S 2013 Development of a three-dimensional finite-element model for high-temperature superconductors based on the H-formulation Workshop 'CHATS Appl. Supercond. 2011' 53 142-7

[29] Bossavit A 1988 Whitney forms: a class of finite elements for three-dimensional computations in electromagnetism IEE Proc. A 135493

[30] Carrier J and Greengard L 1988 A fast adaptive multipole algorithm for particle simulations SIAM J. Sci. Stat. Comput. $9669-86$
[31] Prigozhin L 1997 Analysis of critical-state problems in type-II superconductivity IEEE Trans. Appiled Supercond. 7 3866-73

[32] Pardo E, Šouc J and Frolek L 2015 Electromagnetic modelling of superconductors with a smooth current-voltage relation: variational principle and coils from a few turns to large magnets Supercond. Sci. Technol. 28044003

[33] Prigozhin L 1998 Solution of thin film magnetization problems in type-II superconductivity J. Comput. Phys. 144 180-93

[34] Amemiya N, Sato S and Ito T 2006 Magnetic flux penetration into twisted multifilamentary coated superconductors subjected to ac transverse magnetic fields J. Appl. Phys. 100123907

[35] Morandi A and Fabbri M 2015 A unified approach to the power law and the critical state modeling of superconductors in 2D Supercond. Sci. Technol. 28024004

[36] Pardo E and Kapolka M 2017 3D computation of non-linear eddy currents: variational method and superconducting cubic bulk J. Comput. Phys. 344 339-63

[37] Nii M, Amemiya N and Nakamura T 2012 Three-dimensional model for numerical electromagnetic field analyses of coated superconductors and its application to Roebel cables Supercond. Sci. Technol. 25095011

[38] Stenvall A, Lahtinen V and Lyly M 2014 An H-formulationbased three-dimensional hysteresis loss modelling tool in a simulation including time varying applied field and transport current: the fundamental problem and its solution Supercond. Sci. Technol. 27104004

[39] Ruehli A E 1974 Equivalent circuit models for threedimensional multiconductor systems IEEE Trans. Microw. Theory Tech. 22 216-21

[40] Wang T et al 2015 Analyses of transient behaviors of noinsulation REBCO pancake coils during sudden discharging and overcurrent IEEE Trans. Appl. Supercond. 254603409

[41] Dular P, Hody J-Y, Nicolet A, Genon A and Legros W 1994 Mixed finite elements associated with a collection of tetrahedra, hexahedra and prisms IEEE Trans. Magn. 30 2980-3

[42] Masserey A, Rappaz J, Rozsnyo R and Swierkosz M 2005 Numerical integration of the three-dimensional green kernel for an electromagnetic problem J. Comput. Phys. 205 48-71

[43] Bebendorf M 2000 Numer. Math. 86565

[44] Börm S and Grasedyck L 2005 Hybrid cross approximation of integral operators Numer. Math. 101 221-49

[45] Nguyen T-S, Guichon J-M, Chadebec O, Meunier G and Vincent B 2012 An independant loop search algorithm for solving inductive PEEC large problems Prog. Electromagn. Res. M 23 53-63

[46] Paul W, Hu D and Baumann T 1991 Voltage-current characteristic between $10^{-13} \mathrm{Vcm}^{-1}$ and $10^{-3} \mathrm{Vcm}^{-1}$ of BSCCO and time decay of the magnetization Physica C 185-189 2373-4

[47] Rhyner J 1993 Magnetic properties and AC-losses of superconductors with power law current-voltage characteristics Physica C 212 292-300

[48] Uglietti D, Kitaguchi H, Choi S and Kiyoshi T 2009 Angular dependence of critical current in coated conductors at $4.2 \mathrm{~K}$ and magnet design IEEE Trans. Appl. Supercond. 19 2909-12

[49] G2ELab 2018 MIPSE : Modeling of Interconnected Power SystEms http://www.g2elab.grenoble-inp.fr/en/platforms/ mipse-modeling-of-interconnected-power-systems-1 (accessed 6 February 2019)

[50] HTS Modeling Workgroup: benchmarks http://htsmodelling. com/?page_id=2 (accessed 6 February 2019)

[51] Sirois F and Roy F 2007 Computation of 2D current distribution in superconductors of arbitrary shapes using a new semi-analytical method IEEE Trans. Appl. Supercond. 17 3836-45 
[52] Morandi A, Perini E, Giunchi G and Fabbri M 2011 Numerical analysis and experimental measurements of magnetic bearings based on $\mathrm{MgB}_{2}$ hollow cylinders IEEE Trans. Appl. Supercond. 21 1460-3

[53] Ainslie M D and Fujishiro H 2015 Modelling of bulk superconductor magnetization Supercond. Sci. Technol. 28 053002

[54] Nishijima G, Tsuchiya Y, Kitaguchi H, Nishimura T and Kato T $2013 I_{\mathrm{c}}-B-T$ evaluation for high- $T_{\mathrm{c}}$ superconductors in pressurized/depressurized liquid nitrogen IEEE Trans. Appl. Supercond. 23 8000703-8000703

[55] Benkel T et al 2016 REBCO performance at high field with low incident angle and preliminary tests for a 10-T insert IEEE Trans. Appl. Supercond. 264302705
[56] TAPESTAR-Family: quality management for coated conductors https://www.theva.com/products/\#tapestar (accessed 6 February 2019)

[57] Senatore C, Barth C, Bonura M, Kulich M and Mondonico G 2016 Field and temperature scaling of the critical current density in commercial REBCO coated conductors Supercond. Sci. Technol. 29014002

[58] Samoilenkov S et al 2016 Customised 2G HTS wire for applications Supercond. Sci. Technol. 29024001

[59] Escamez G, Sirois F, Badel A, Meunier G, Ramdane B and Tixador P 2016 Numerical impact of using different $E-J$ relationships for $3 \mathrm{D}$ simulations of AC losses in $\mathrm{MgB}_{2}$ superconducting wires IEEE Trans. Magn. 52 7402904 